\title{
ORGANISMAL RESPONSE TO OXIDATIVE
} STRESS

\author{
Tin Klanjscek ${ }^{a, b}$, Erik B. Muller ${ }^{a}$, Roger M. Nisbet ${ }^{a}$
}

May 23, 2016

\footnotetext{
${ }^{a}$ University of California Santa Barbara, Santa Barbara, CA, USA

${ }^{b}$ Ruder Boskovic Institute, Zagreb, Croatia

e-mail of the corresponding author: tin@irb.hr
}

\begin{abstract}
Biological feedbacks play a crucial role in determining effects of toxicants, radiation, and other environmental stressors on organisms. Focusing on reactive oxygen species (ROS) that are increasingly recognized as a crucial mediator of many stressor effects, we investigate how feedback strength affects the ability of organisms to control negative effects of exposure. We do this by developing a general theoretical framework for describing effects of a wide range of stressors and species. The framework accounts for positive and negative feedbacks representing cellular processes: (i) production of ROS due to metabolism and the stressor, (ii) ROS reactions with cellular compounds that cause damage, and (iii) cellular control of both ROS and damage. We suggest functional forms that capture generic properties of cellular control mechanisms constituting the feedbacks, assess stability of equilibrium states in the resulting models, and investigate tipping points at which cellular control breaks down causing unregulated increase of ROS and damage. Depending on the chosen functional forms, the
\end{abstract}


38

40

41

42

43 9 0

models can have zero, one, or two positive steady states; except in one singular case, the steady state with lowest values of ROS and damage is locally stable. We found two types of tipping points: those induced by changes in the parameters (including the stressor intensity), and those induced by the history of exposure, i.e. ROS and damage levels. The relatively simple models effectively describe several patterns of cellular responses to stress, such as the covariation of ROS and damage, the break-down of cellular control leading to explosion of ROS and/or damage, increase in damage even when ROS is (near)-constant, and the effects of exposure history on the ability of the cell to handle additional stress. The models quantify dynamics of cellular control, and could therefore be used to estimate the metabolic costs of stress and help integrate them into models that use energetic considerations to model organism's response to the environment. Although developed with unicellular organisms in mind, our models can be applied to all multicellular organisms that utilize similar feedbacks when dealing with stress.

\subsection{Keywords}

reactive oxygen species (ROS), cellular damage, organismal response to stress, modeling damage repair, stability, runaway ROS and damage. 


\section{${ }_{45} 1$ Introduction}

The cell's attempt to reduce deleterious effects of ROS such as membrane, DNA, and protein damage by regulating levels of both ROS and damage. Exposure to environmental stressors, including toxic exposure to transition metal ions and engineered nano-particles (ENPs), results in greatly enhanced ROS production rates that, in turn, require increase in control of ROS and damage. Accordingly, several empirical studies in eco- and nano-toxicology report simultaneous measurements of ROS and of some proxy for cellular damage (e.g. Nel et al. 2006, Priester et al. 2009, Priester et al. 2012, Ivask et al. 2014, Kaweeteerawat et al. 2015). These studies and others, found distinct correlation between ROS, the proxies for damage, and (for bacteria) reduction in population growth.

With toxic effect models, we have previously demonstrated that simultaneous measurements of ROS and of responses to ROS in bacteria, exposed to cadmium and cadmium-based ENPs, aid in elucidating the potential modes of toxicant action at the suborganismal to population levels of organization (Klanjscek et al. 2012; Klanjscek et al. 2013). Our previous work was based on Dynamic Energy Budget (DEB) theory (Kooijman, 2010), a unifying and relatively abstract modelling framework with many applications in ecotoxicology (e.g. Jager and Zimmer 2012, Martin et al. 2013, Jager et al. 2014a, Jager et al. 2014b). The resulting models were able to use the patterns of ROS and damage accumulation at a constant exposure to predict toxicity, but were of limited use for modeling dynamics of repairable damage, and investigating key features of cellular control.

Known cellular control mechanisms instigate negative feedbacks that reduce ROS and damage levels. ROS levels are reduced using antioxidant enzymes such as catalases, glutathionperoxidases and peroxiredoxins, and non-enzymatic radical scavengers (e.g. Bannister et al. 1987, Jamet et al. 2003, Giorgio et al. 2007, Culotta and Daly 2013), while damage is controlled using repair pathways ranging from protein (see Chondrogianni et al. (2014) for a review) to DNA repair (e.g. Gros et al. 2002, Zahradka et al. 2006). The negative feedbacks are subject to physiological and biochemical constraints including energy and materials available to them. The constraints may be 
72 the reason why many studies (e.g. Priester et al. 2009, Kriško and Radman 2010) note a fairly

73

74

75

76 77 and damage leading to rapid mortality.

78

79

80

81

82

83

84

small increase in ROS and/or damage for a range of exposure levels, followed by a large increase

in both for a relatively small additional increase in exposure: for sufficiently high ROS and/or damage production rates, the negative feedbacks may not be able to keep up, the control breaks, and ROS and damage experience a runaway, i.e. an accelerated and unbounded increase of ROS

To account for these biomolecular defense and repair mechanisms, and their breakage, we develop simple models to explore the effects of positive and negative feedbacks on the dynamics of damage and damage-inducing compounds in response to environmental stressors. We investigate how particular control mechanisms affect the long-term dynamics, and identify contexts that may cause a runaway increase in ROS and/or damage that would lead to rapid mortality. We discuss potential applications and limitations of the new models, and analyze some of the ways in which duration of experiments and combinations of stressor intensities might affect results. 


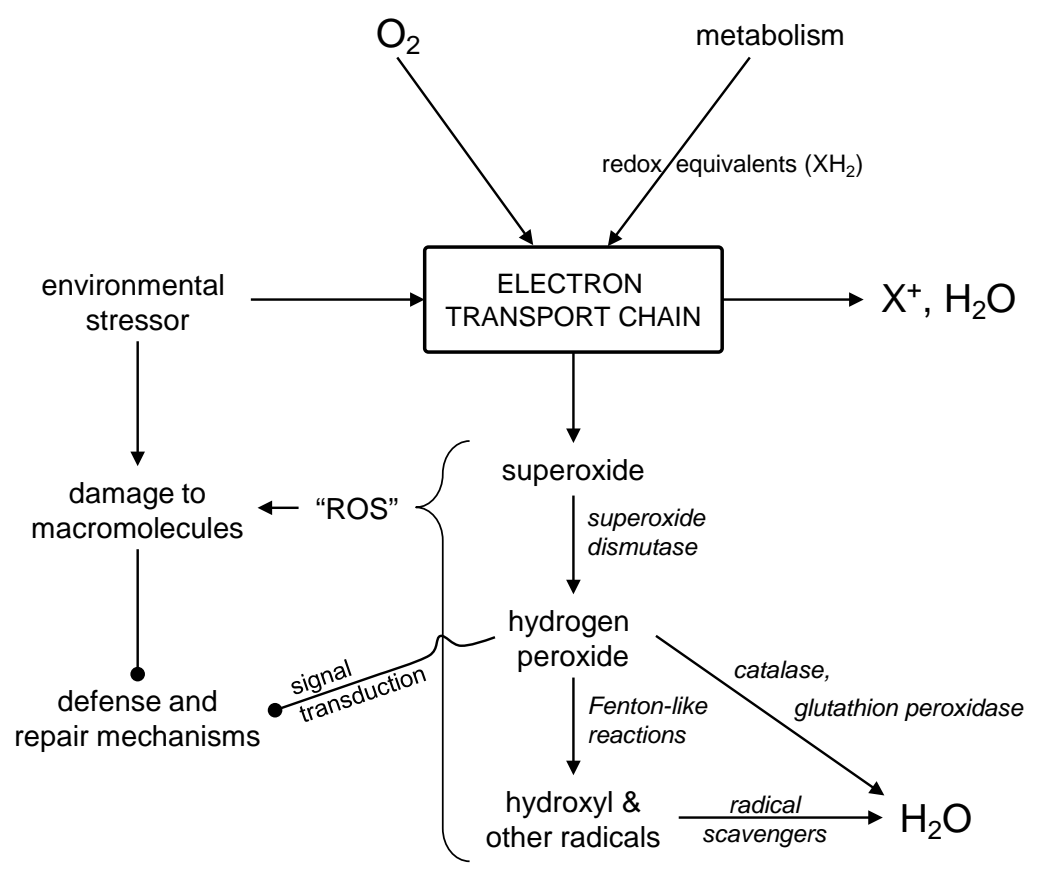

Figure 1: Outline of oxidative stress network that inspired the development of our models. Metabolism and various environmental stressors give rise to the production of compounds with high oxidative potential, such as radicals and hydrogen peroxide. For example, reduced metabolites $\left(\mathrm{XH}_{2}\right)$ donate electrons to components of electron transport chains that, under the influence of the environmental stressor, achieve relatively high redox potentials and thereby facilitate the production of superoxide radicals. This initiates a cascade of reactions involving reactive oxygen species (ROS) that are mediated by enzymes such as superoxide dismutase, catalase, glutathion peroxidase and peroxiredoxins, and more haphazardly by metalloenzymes (Fenton-like reactions in the figure). These reactions do not directly result in cellular damage. However, ROS may spontaneously react with cellular compounds such as lipids, DNA and proteins. These reactions can inflict damage. Damage can also be inflicted directly by environmental stressors such as ionizing radiation and transition metals. Intermediate steps in the radical cascade, as well as actual damage, can serve as signals for up-regulation of defense and/or repair mechanisms (among other mechanisms not dealt with here, such as degenerative and developmental processes). Damaged molecular machinery may not be as efficient in controlling ROS production (e.g. damaged mitochondria leak more ROS).

Our models aim at generality, but they have been inspired by specific mechanisms (see Figure 1). Metabolism and environmental stressor impact yield a variety of damage-inducing compounds with different physicochemical properties and damaging potentials. Given the prevalence of ROS as damage-inducing compounds in the literature (see Imlay 2003 for a review on pathways of oxidative damage), we will use these terms interchangeably. In order to keep our representation of the 
107

108

109

110

111

112

113

114

115

116

117

118

119

120

dynamics of reactive compounds tractable, we assume that each physiological process (metabolism and impact of each distinct stressor) generates a set of reactive compounds whose relative proportions are approximately constant. This enables us to aggregate compounds with significant oxidative potential into a single, abstract "generalized" entity, i.e. damage-inducing compounds or ROS. Similarly, there are many forms of damage, but for the present work we aggregate these forms into a generalized damage compound. We denote concentrations of ROS and damage in a cell by $Z$ and $D$, respectively. We leave the term "concentration" deliberately vague; the actual units can be chosen to best fit a particular application.

In most of what follows, we assume that - given sufficient time, energy, and material resources - damage can be repaired. In reality, some damage is irreparable. Examples of irreparable damage include permanent loss of information through DNA damage and/or loss of epigenetic information, proteins wrongly produced because of permanent loss of information, as well as irreparable organ and/or tissue damage in multicellular organisms. We discuss the implication of irreparable damage in the Discussion. 


\subsection{Choice of variables and balance equations}

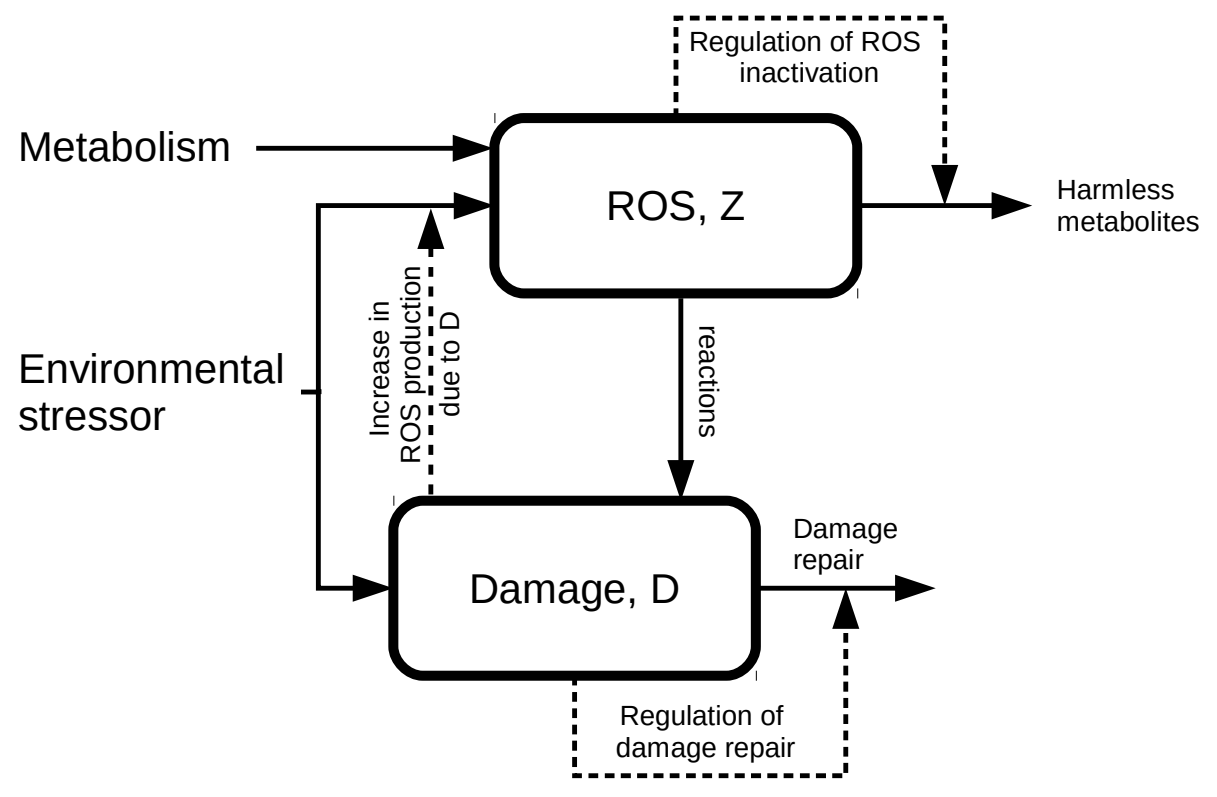

Figure 2: Model structure. Metabolism and environmental stressor produce ROS. In part, ROS is ultimately reduced to harmless compounds, such as water, through controlled reactions without causing damage to the cell. However, ROS also inflicts damage through spontaneous, uncontrolled reactions with cellular compounds. Damage is reduced by regulated repair mechanisms, while non-repaired damage may enhance ROS production.

Figure 2 describes the basic relationships of the processes determining the dynamics of ROS and damage: environmental stressors and metabolism produce ROS (of concentration $Z$ ) that, unless inactivated, can produce damage (of concentration $D$ ). Damage may enhance ROS production, but can also be repaired. The rate of ROS inactivation, as well as the rate of damage repair, can be regulated by the cell. In general terms, the system can be described by the following mass balance equations:

$$
\begin{aligned}
\frac{d Z}{d t} & =j_{Z, \text { prod }}-j_{Z, \text { out }}-\delta_{Z}^{G} \\
\frac{d D}{d t} & =j_{D, \text { prod }}-j_{D, \text { out }}-\delta_{D}^{G},
\end{aligned}
$$

where $\delta_{Z, D}^{G}$ accounts for dilution, i.e. changes in concentrations of $Z$ and $D$ due to growth or shrinking of the organism. Fluxes $j_{\text {prod }}$ and $j_{\text {out }}$ represent, respectively, production and loss rates. 
For the current presentation, we assume that organismal growth (or shrinking) is a slow process relative to the dynamics of ROS and damage and set the dilution terms to zero $\left(\delta_{Z, D}^{G}=0\right)$, but note that growth may need to be considered in some applications (see Klanjscek et al. (2012, 2013)). The production and loss rates are discussed below.

The ROS production flux $\left(j_{z, \text { prod }}\right)$ is assumed to be an increasing function of metabolically induced ROS, stressor-induced ROS, and ROS production enhanced by cellular damage. We compound the first two sources of ROS into a production term, $P_{Z}$ :

$$
P_{Z}=P_{0}+\gamma_{Z S} S
$$

where $P_{0}$ and $S$ represent respectively the metabolic ROS production flux and stressor intensity. The relationship between $P_{Z}$ and $S$ will depend on the actual stress mechanism; for this study, we assume a linear functional dependence where the coefficient $\gamma_{Z S}$ quantifies how much ROS is produced given some stressor intensity.

Damage can affect ROS production in a myriad of ways. We consider a linear combination of multiplicative and additive effects:

$$
j_{Z, p r o d}=\left(1+g_{Z D} D\right) P_{Z}+\gamma_{Z D} D
$$

where $g_{Z D}$ is the multiplicative, and $\gamma_{Z D}$ the additive damage interaction coefficient. The multiplicative term $\left(g_{Z D} P_{Z} D\right)$ accounts for increases in ROS production due to metabolic inefficiencies resulting from damage, and the additive term $\left(\gamma_{Z D} D\right)$ accounts for other damage-related sources of ROS.

The ROS removal flux $\left(j_{Z, o u t}\right)$ is a consequence of reactions with cellular components. Some ROS is removed by controlled reactions without creating damage. ROS not removed by the controlled reactions can react with other cellular components and cause damage. Since at any given time the number of ROS molecules is minuscule compared to the number of potential target sites (Giorgio et al.2007), spontaneous ROS reactions are limited by the concentration of ROS only, 
and the can be assumed to be proportional to the ROS concentration. The total ROS removal flux is thus the sum of the two, controlled and spontaneous, reaction fluxes:

$$
j_{Z, \text { out }}=k_{Z} Z+R_{Z}
$$

where $k_{Z}$ is a rate coefficient for the spontaneous flux, and $R_{Z}$ represents the flux resulting from controlled reactions (see Appendix A for details).

The damage production flux $\left(j_{D, p r o d}\right)$ has two components: direct, stress-induced damage production, $P_{D}$, and damage created by spontaneous ROS reactions. The direct stressor-induced damage production term is represented by

$$
P_{D}=\gamma_{D S} S
$$

where the damage stress coefficient, $\gamma_{D S}$, quantifies the yield of damage due to direct stressor action. For simplicity, we assume that the damage yield from oxidative stress is constant, $y_{D}$; the total damage creation flux is then:

$$
j_{D, p r o d}=y_{D} k_{Z} Z+P_{D}
$$

The damage removal flux $\left(j_{D, \text { out }}\right)$ accounts for all cellular repair processes, including the metabolic turnover of damaged macromolecules. We assume that spontaneous damage repair is negligible, and that all damage repair is regulated:

$$
j_{D, \text { out }}=R_{D}
$$

where $R_{D}$ represents regulated damage repair.

The regulatory networks exerting the control of damage repair and of ROS are context-specific and can be extremely complex, so we approximate their dynamics through simple, stylized negative feedback loops (see Zhang and Andersen (2007) and Drengstig et al. (2012) for examples). Such 
175

176

177

178

179

180

181

182

183

184

185

186

general feedback loops have at minimum one controller variable describing the controller compound, and one controlled variable describing the controlled compound. The rate of change of each controller $X$ controlling a variable $Y$ is governed by a balance equation

$$
\frac{d X}{d t}=j_{X, p r o d}-j_{X, o u t} .
$$

We assume that:

- $j_{X, p r o d}$ can be represented by a Michaelis-Menten type saturating function, $j_{X, p r o d}=\frac{v_{X} Y}{K_{X}+Y}$, because we expect:

- $j_{X, p r o d}$ is an increasing function of $Y$ : production rates of the controllers increase as ROS and damage concentrations increase (for example, cells up-regulate defenses against ROS as ROS concentrations increase (Giorgio et al. 2007), e.g. by initiating the SOS gene pathway when damage accumulates (Friedman et al. 2005), and

- $j_{X, \text { prod }}$ is a saturating function of $Y$ : the cellular capacity to produce controller compounds is bounded, i.e. there is an upper limit to the rate of controller production.

- $j_{X, \text { out }}$ is an increasing function of $X$ : controllers are inactivated due to cellular turnover and to interactions with the controlled compounds; we assume a simple linear, passive inactivation: $j_{X, \text { out }}=k_{X} X$.

We introduce two additional variables, $E$ and $A$, to represent respectively the concentrations of the compounds controlling ROS and damage. We consider two types of interaction between the controller compounds $(E, A)$ and the controlled compounds $(Z, D)$ : bilinear and saturating Michaelis-Menten kinetics (see Table 1 and Appendix A for details). Dynamics of $Z$ and $D$ are of special importance because there are many experimental contexts where ROS and some measure of damage play a role and where data are available. We therefore focus on dynamics of $Z$ and $D$ by assuming the transients of controllers are not important $(d E / d t=d A / d t=0)$, which results in implicit representations of control $\left(R_{Z}\right.$ and $\left.R_{D}\right)$ listed in Table 1, with details of their derivation in 
Appendix A. This assumption may not hold if a sudden, acute exposure causes massive increase in ROS and/or damage in a time span much shorter than the response time of the controls.

Table 1: Summary of functional forms of ROS and damage production fluxes, ROS inactivation, and damage repair. Extremes cases of the production flux are: additive $\left(g_{Z D}=0, \gamma_{Z D}>0\right)$, and multiplicative $\left(g_{Z D}>0, \gamma_{Z D}=0\right)$. Repair of $\operatorname{ROS}(Z)$ and damage $(D)$ are regulated by control variables $E$ and $A$, respectively; interaction terms and the resulting implicit forms are listed. Compound control parameters are used for simplicity: $v_{Z}=g_{Z} v_{E} / k_{E}$, and $v_{D}=g_{D} v_{A} / k_{A}$, where $v_{E}$ and $v_{A}$ are the maximum production rates of $E$ and $A ; g_{Z}$ and $g_{D}$ quantify control strength, while $k_{E}$ and $k_{A}$ are inactivation rates of the controller. $K_{E}$ and $K_{A}$ are half-saturation constants for production of $E$ and $A ; K_{Z}$ and $K_{D}$ are half-saturation constants for control of $Z$ and $D$.

\begin{tabular}{|c|c|}
\hline \multicolumn{2}{|c|}{ ROS production, $j_{Z, \text { prod }}$} \\
\hline additive & $P_{Z}+\gamma_{Z D} D$ \\
\hline multiplicative & $\left(1+g_{Z D} D\right) P_{Z}$ \\
\hline
\end{tabular}

\begin{tabular}{|c|c|c|}
\hline \hline \multicolumn{2}{|c|}{ controlled ROS inactivation, $R_{Z}$} \\
\hline \multicolumn{2}{|c|}{ interaction term } & implicit form \\
\hline linear & $g_{Z} E Z$ & $\frac{v_{Z} Z^{2}}{K_{E}+Z}$ \\
\hline saturating & $g_{Z} E Z \frac{K_{Z}}{K_{Z}+Z}$ & $\frac{v_{Z} Z^{2}}{\left(K_{E}+Z\right)\left(1+Z / K_{Z}\right)}$ \\
\hline \hline
\end{tabular}

\begin{tabular}{|c|c|c|}
\hline \multicolumn{3}{|c|}{ Damage repair, $R_{D}$} \\
\hline \multicolumn{2}{|c|}{ interaction term } & implicit form \\
\hline linear & $g_{D} A D$ & $\frac{v_{D} D^{2}}{K_{A}+D}$ \\
\hline saturating & $g_{D} A D \frac{K_{D}}{K_{D}+D}$ & $\frac{v_{D} D^{2}}{\left(K_{A}+D\right)\left(1+D / K_{D}\right)}$ \\
\hline
\end{tabular}

\section{$2.2 \quad$ Steady state analysis}

The steady state analysis, performed using implicit form of ROS and damage control $\left(R_{Z}\right.$ and $R_{D}$ ), is greatly facilitated by quantification of feedbacks by their strength. We define the feedback 
213

strength, $\Phi_{X Y}$, of a state variable $Y$ on dynamics of state variable $X$ as:

$$
\Phi_{X Y}=\frac{\partial d X / d t}{\partial Y}
$$

214

215

216

217

218

219

Our system has two state variables and, therefore, four possible feedbacks with the associated strengths: (i) a negative feedback that increases ROS removal in response to ROS concentration $\left(\Phi_{Z Z}^{-}=-\left(k_{Z}+\partial R_{Z} / \partial Z\right)\right)$, (ii) a positive feedback that increases damage production in response to ROS concentration $\left(\Phi_{D Z}^{+}=y_{D} k_{Z}\right)$, (iii) a positive feedback that increases ROS production in response to damage $\left(\Phi_{Z D}^{+}=\partial j_{Z, p r o d} / \partial D\right)$, and (iv) a negative feedback that increases damage repair in response to damage $\left(\Phi_{D D}^{-}=-\partial R_{D} / \partial D\right)$. Feedbacks (i) and (iii) are considered positive because they increase $Z$ and/or $D$, and feedbacks (ii) and (iv) are negative because they reduce $Z$ and $D$.

Seven model variants with different combinations of ROS production, ROS control, and damage control were analyzed. Depending on the model variant and the feedback strengths, the system may have had zero, one, or two steady states. An overview of the six model variants and results for which ROS and/or damage control are linear are included in Table 2. The model variant in which both ROS and damage control are saturating does not qualitatively differ from the models with saturating damage control, so was omitted from the overview.

In Appendix $\mathrm{C}$ we show that for each of our six model variants, there is a unique positive stable equilibrium (UPSE) if and only if the combined effect of the negative feedbacks is stronger than those of the positive feedbacks as $Z$ and $D$ approach infinity:

$$
\lim _{Z, D \rightarrow \infty} \Phi_{Z D}^{+} \Phi_{D Z}^{+}<\lim _{Z, D \rightarrow \infty} \Phi_{Z Z}^{-} \Phi_{D D}^{-}
$$

The left-hand side of (11) can be interpreted as susceptibility to stress, and the right-hand side as resistance to stress; the condition (11) then simply states that there is a UPSE whenever resistance is greater than susceptibility to stress. Functional forms of the feedbacks and UPSE existence conditions for each of the six model variants are summarized in Table 2.

When effects of damage on ROS are multiplicative and damage repair $\left(R_{D}\right)$ is linear, the UPSE 


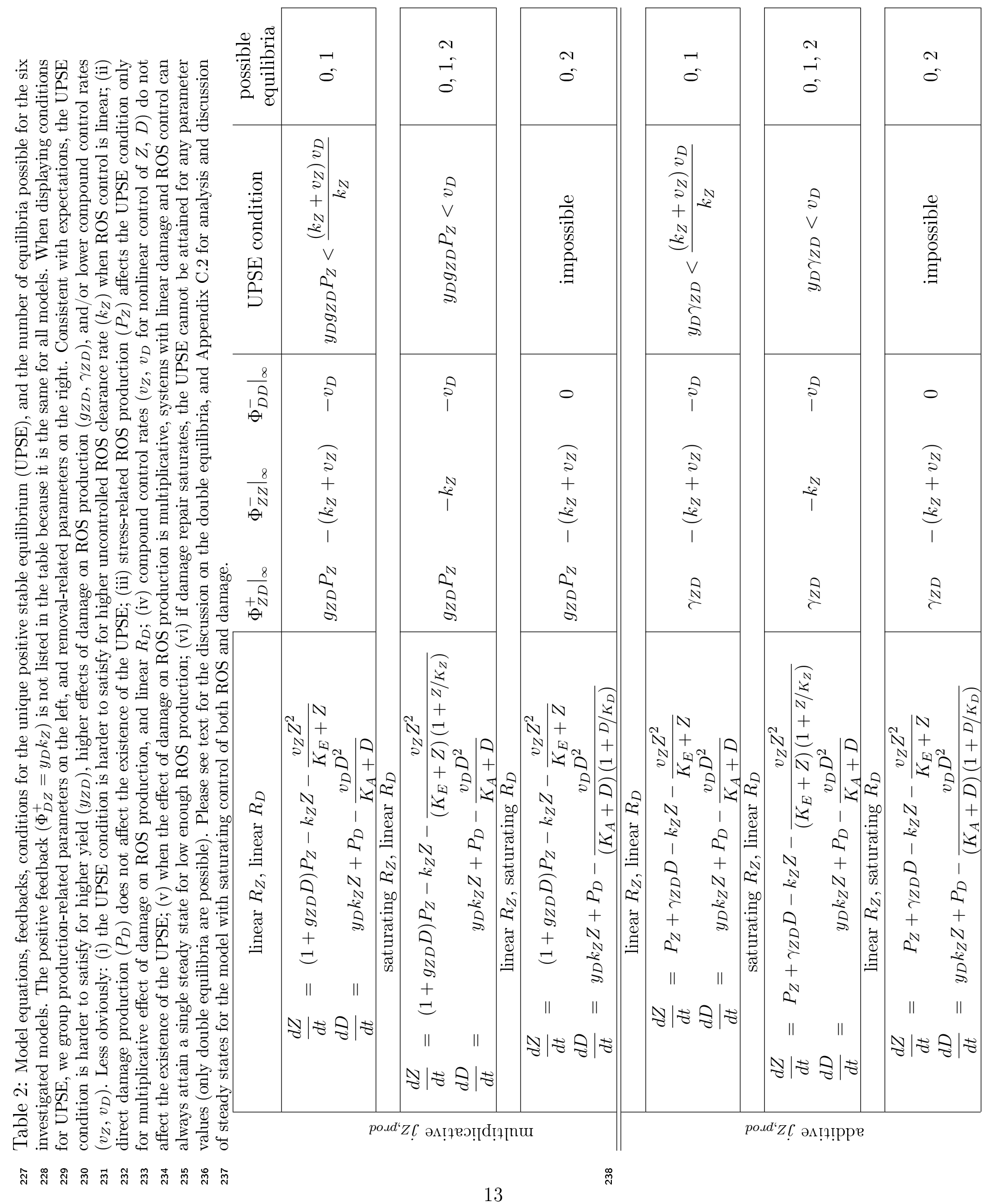


condition can always be satisfied for low enough stressor intensities, i.e. when $P_{Z}<P_{Z}^{\Phi}$ where:

$$
P_{Z}^{\Phi}= \begin{cases}\frac{\left(k_{Z}+v_{Z}\right) v_{D}}{k_{Z} y_{D} g_{Z D}} & \text { if ROS control is linear } \\ \frac{v_{D}}{y_{D} g_{Z D}} & \text { if ROS control is saturating. }\end{cases}
$$

When effects of damage on ROS are additive, the UPSE condition does not depend on stressor intensities. For saturating $R_{D}$, a UPSE is never possible, so the system can never have a UPSE. Note that both types of stressor effects $\left(P_{Z}\right.$ and $\left.P_{D}\right)$ affect the equilibrium values of ROS and damage, even if they cannot affect the conditions for the existence of a UPSE.

When UPSE condition is satisfied, the organism's cellular control mechanisms are, in principle, able to cope with any amount of ROS and damage. If the condition is not satisfied, there are two possibilities:

1. The system has two equilibria: one stable with lower $Z$ and $D$ values, and one unstable with higher $Z$ and $D$ values. If $Z$ and $D$ are lower than the values for the unstable equilibrium, the system approaches the stable equilibrium, and ROS and damage are controlled. If $Z$ and $D$ increase above the values for unstable equilibrium, ROS and damage increase indefinitely. Only models with saturating control can have two equilibria.

2. There are no equilibria. ROS and damage increase indefinitely at all stressor intensities.

\section{$2.3 \quad$ Tipping points}

We use the term "tipping point" to characterize a transition from a stable state to runaway dynamics by which we mean continuous, unbounded increase over time of $Z$ and $D$. We distinguish between two types of tipping points.

Type 1 tipping point (TP1) is caused by changes in parameters and/or forcing (stressor intensity), and can appear in systems with both linear and saturating control. In systems with linear ROS control and damage repair (linear $R_{Z}$ and $R_{D}$ ), any changes in parameters or forcing that leads to invalidation of the UPSE condition causes a transition from a system with a unique stable 
equilibrium to a system with runaway dynamics (see the steady state analysis in Section 2.2). When the control is saturating, and the system has two equilibria, change in parameters or forcing can cause a saddle node bifurcation in which the two equilibria approach each other, combine into a single neutral equilibrium, and then disappear causing runaway dynamics (see Appendix C).

Type 2 tipping point (TP2) only exists in two-equilibria systems. The tipping point is caused by high values of $Z$ and $D$ resulting from high initial values or large transients. In two-equilibrium systems, the organism is able to control ROS and damage indefinitely if the initial values of $Z$ and $D$ are low enough; if $Z$ and/or $D$ increase above a threshold (see Appendix C), positive feedbacks take over, control mechanisms are overwhelmed, and ROS $(Z)$ and damage $(D)$ increase indefinitely at an accelerating rate.

We illustrate these possibilities using a reduced model that follows what is arguably the most important state variable - damage. Recognizing that ROS and control typically operate on much shorter time scales than damage, we separate the time scales and set $d Z / d t=0$. Expressing $R_{Z}$ in terms of $Z$, and solving (1) for the quasi-equilibrium value of $Z, Z^{*}$, gives:

$$
Z^{*}=\xi\left(D, P_{Z}\right)
$$

where the function $\xi$ normally has to be evaluated numerically. Inserting $Z^{*}$ into (2) gives just one - albeit complicated - ODE describing the slow dynamics of a general form:

$$
\frac{d D}{d t}=y_{D} k_{Z} \xi\left(D, P_{Z}\right)+P_{D}-R_{D}
$$

For illustration, we choose a model with $P_{D}=0$ (no direct damage production), multiplicative $j_{Z, \text { prod }}$, saturating $R_{Z}$, and linear $R_{D}$. The choice enables us to investigate the widest range of possible dynamics, as well as analyze the tipping points as a function of stress. We calculated a "canonical" parameter set (gu)estimated from a number of different studies. These parameters do not represent any one system, but their foundation in data suggests that we explored "plausible" regions of parameter space. Details and a summary of all state variables and parameter values are 
in Appendix B.

Increase in stress can cause two types of bifurcation: from an UPSE to a double equilibrium at $P_{Z}=P_{Z}^{\Phi}$ (making TP2 possible), and the transition from a two-equilibria system to a runaway at $P_{Z}=P_{Z}^{C}(\mathrm{TP} 1)$. The critical stress $P_{Z}^{\Phi}$ was calculated by equating the limits in (11) and solving for $P$, and $P_{Z}^{C}$ was estimated numerically. Since $P_{Z}^{\Phi}$ is a linear function of $y_{D}, \gamma_{Z D}$, and $1 / v_{D}$ (see (12) for saturating ROS control), increasing $y_{D}$ and $\gamma_{Z D}$, or reducing $v_{D}$ can cause the same transition to a double-equilibrium dynamics as increasing $P_{Z}$.

Three regions delineated by the UPSE condition and the saddle-node bifurcation are illustrated in Figure 3, with further details and trajectories shown in Figure 4. Trajectories $D(t)$ with $P_{Z}<P_{Z}^{\Phi}$, as expected for a unique positive stable equilibrium (UPSE), eventually attain a finite equilibrium value of $D$ for all considered values of initial damage $D(0)$; if an additional stressor temporarily increases $D$, the organism will be able to repair the excess damage once the additional stress stops. The exact value of the equilibrium in our example depends on the stress intensity, but is always less than $D_{\Phi}$, the equilibrium value of $D$ for $P_{Z}^{\Phi}\left(D_{\Phi}=0.248\right.$ in Figure 4$)$.

As exposure $\left(P_{Z}\right)$ increases, non-linear effects become increasingly important, and the system has two equilibria. The lower equilibrium is stable; the higher one $\left(D_{U}\right)$ is unstable. The organism will still able to control additional short-term stress, but not as effectively as for $P_{Z}<P_{Z}^{\Phi}$ : if damage due to the additional stressor increases above $D_{U}$, the organism will not be able to return to any equilibria even if the additional stressor disappears. The higher the value of $P_{Z}$, the lower the range of damage for which the organism can successfully accommodate additional stress: the stable equilibrium increases, while $D_{U}$ decreases. Eventually, the two equilibria overlap for $P_{Z}=P_{Z}^{C}$ (saddle node bifurcation point).

The reduced ability to recover from significant damage is especially important when the organism is exposed to multiple and/or time-varying stressors (Figure 5). For example, even small (potentially unknown) additional stress can completely reverse the outcome of an experiment (Figure 5, left plot). The outcome can likewise be affected by small changes in timing of a time-varying (e.g. pulsed) stressor. For example, small changes in the duration of the stress pulses can com- 
pletely reverse the outcome: even if stress is greater than $P_{Z}^{C}$, the organism can repair damage if the pulse is short. If, on the other hand, the duration of the pulse is long enough that damage increases past the unstable equilibrium $\left(D>D_{U}\right)$, the organism cannot repair damage even after the pulsed stress stops (Figure 5, right plot).

The duration of a hypothetical experiment can also affect the perceived critical stress intensity (Figure 6). The model predicts that large increases of damage in short experiments will appear only for stress intensities that are significantly higher than the critical intensity, leading to a significant overestimate of the critical stress intensity and, therefore, potential overestimate of the safe levels of exposure.

The effects of stressors that directly produce damage $\left(P_{D}\right)$ can be understood from the analysis in Appendix C. The analysis shows that $P_{D}$ can cause saddle node bifurcations only. Consequently, stress increasing directly both ROS and damage $\left(P_{Z}, P_{D}>0\right)$ in two-steady state systems causes qualitatively the same effects as the stress directly affecting ROS only $\left(P_{Z}>0, P_{D}=0\right)$; the addition of direct damage production $\left(P_{D}\right)$ only reduces the range of exposure levels for which the two steady states exist, and reduces the ability of the organism to recover from additional damage (reduces the damage and/or ROS necessary for TP2). 


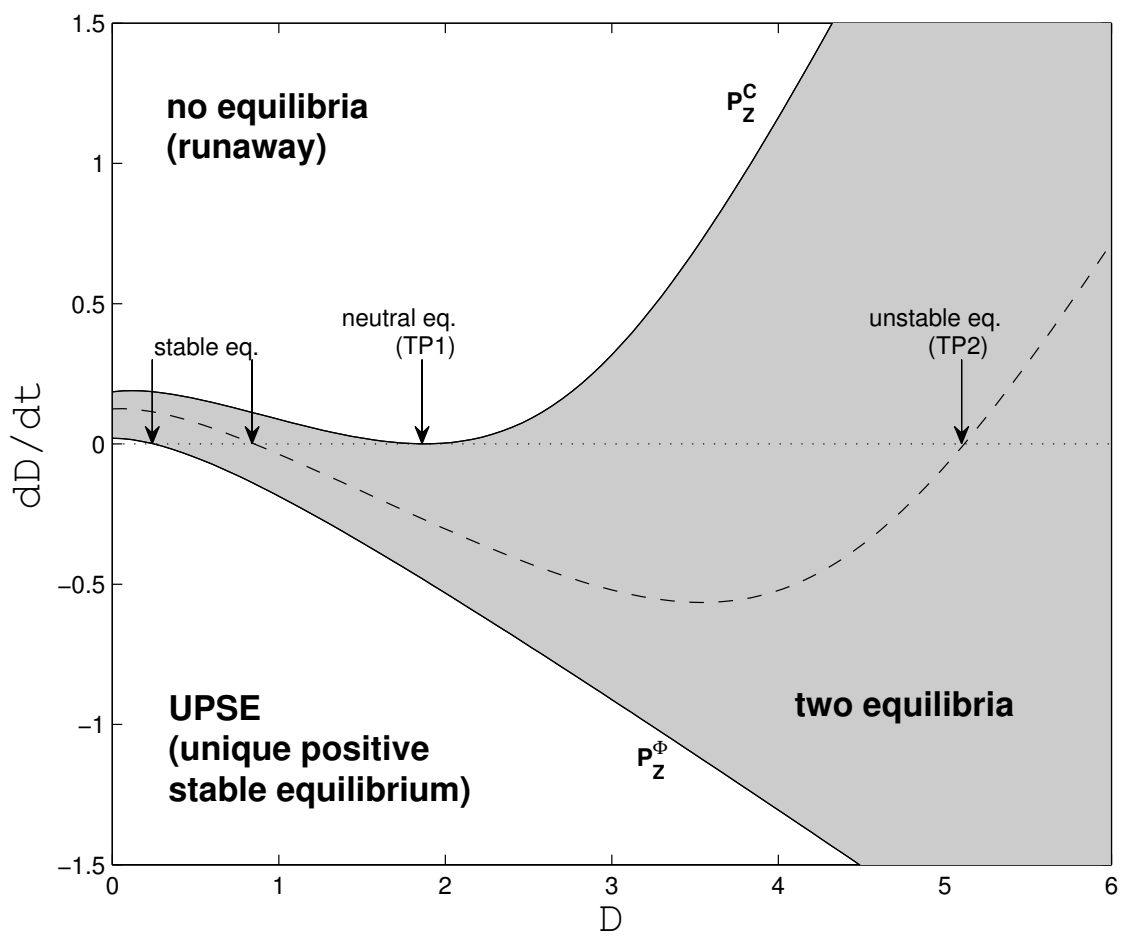

334

Figure 3: Three types of qualitative dynamics for effects of stress on damage production. Rate of change of damage $(d D / d t)$ as a function of damage $(D)$ for $P_{Z}^{\Phi}$ and $P_{Z}^{C}$ (solid lines), and an intermediate value of $P_{Z}$ (dashed line). Intersections of the lines with $y=0$ (dotted line) represent equilibria $(d D / d t=0)$. If the line crosses from positive to negative $d D / d t$ as $D$ increases, the equilibrium is stable; otherwise, it is unstable. Stressor intensities $P_{Z}^{\Phi}$ are characterized by an UPSE with low equilibrium values of $D$ (and, therefore, $Z$ ). As $P_{Z}$ increases above $P_{Z}^{\Phi}$, the stable equilibrium moves to the right (attained for larger $D$ ), the line curves up, and the second, unstable equilibrium is reached for high values of $D$. For $P_{Z}=P_{Z}^{C}$, TP1 is reached (second solid line) with only one neutral equilibrium (at $D=D_{C}=1.86$ ). All stressor intensities higher than $P_{Z}$ result in a runaway. Parameter values listed in Table B.1 used, only $P_{Z}$ varied. 

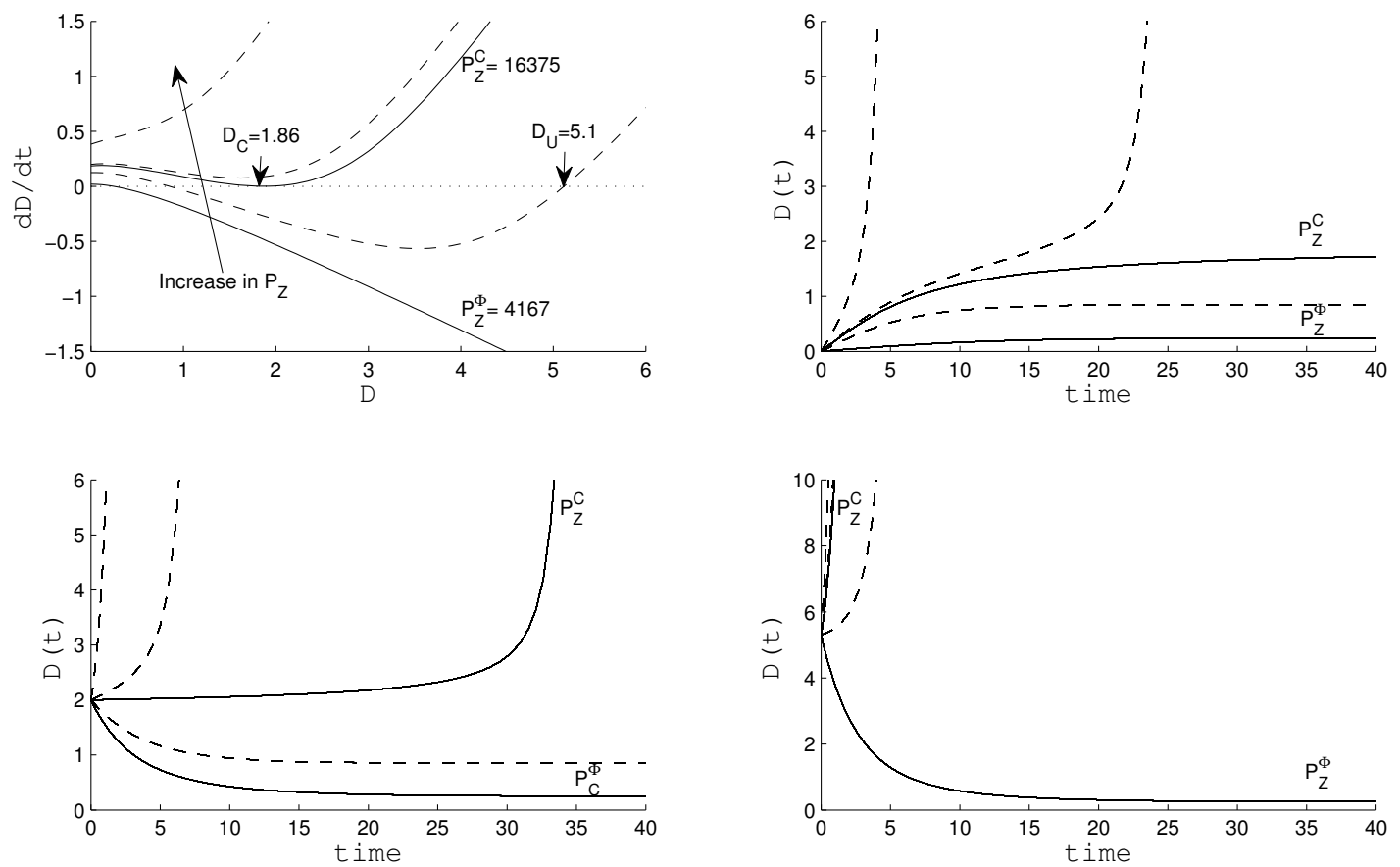

Figure 4: Tipping points and typical trajectories. Top left: rate of change of damage $(d D / d t)$ as a function of damage $(D)$. Consecutive lines represent increasing stressor intensities: $P_{Z}=$ $P_{Z}^{\Phi}, 3.5 P_{Z}^{\Phi}, P_{Z}^{C}, 4 P_{Z}^{\Phi}$, and $4.5 P_{Z}^{\Phi}$. Unstable equilibrium $D_{U}=5.11$ appears for $P_{Z}=3.5 P_{Z}^{\Phi}$. Note that the rate of accumulation of damage for stressor intensities only slightly higher than $P_{C}$ still have a minimum, hence a proximate reduction in the damage increase rate does not necessarily imply successful damage regulation. Other plots: trajectories corresponding to $P_{Z}$ used in the top left plot with different initial values of $D$. Top right: $D(t)$ for $D(0)=0$. Trajectories for $P_{Z}<P_{Z}^{C}$ eventually equilibrate. Bottom left: $D(t)$ for $D(0)=2$. Since $D(0)>D_{C}$, the trajectory for $P=P_{Z}^{C}$ diverges; since $D(0)<D_{U}$, the trajectory for intermediate stressor intensities equilibrates. Bottom right: $D(t)$ for $D(0)=5.3$. Since $D(0)>D_{C}$, all trajectories with $P_{Z}>P_{Z}^{\Phi}$ diverge. Parameter values listed in Table B.1 used, only $P_{Z}$ varied. 


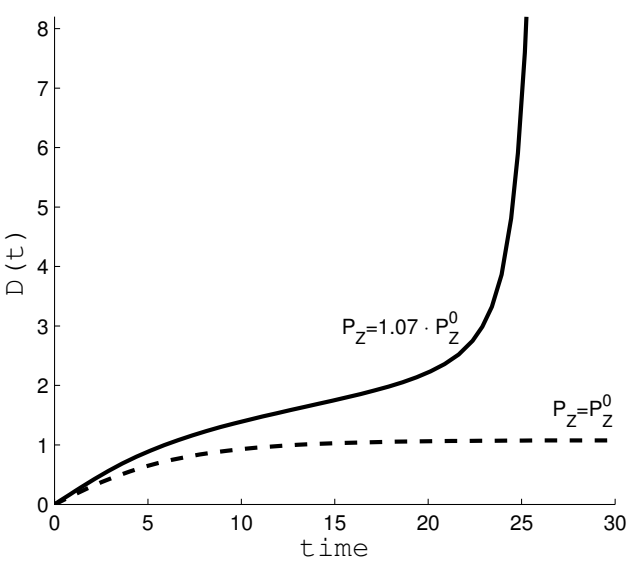

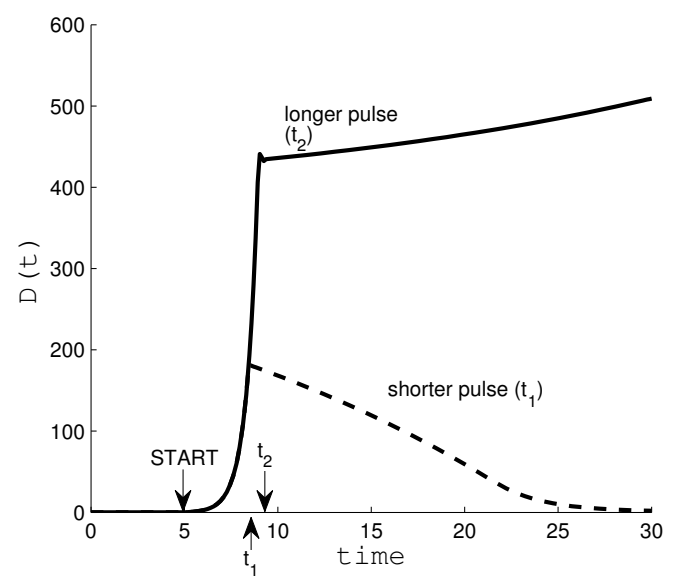

Figure 5: Interpretation of transient dynamics. Left plot: Simulation of an experiment with a target stressor intensity $P_{Z}^{0}=0.95 P_{Z}^{C}$ (dashed line), and an additional stress resulting from the details of the experimental setup. Additional stress is $7 \%$ of target (solid line). Although the organism manages to control damage when exposed to target stressor intensity, $D$ experiences a runaway when the stressors are combined. The closer to $P_{Z}^{C}$ the target stressor intensity is, the smaller the additional stress needed to cause the runaway. Therefore, even small additional stress can completely change the dynamics, especially if target experimental stress $\left(P_{Z}^{0}\right)$ is close to the critical stress $\left(P_{Z}^{C}\right)$. Right plot: Simulations of an experiment with a stress pulse in addition to the background stress. Background stress: $1.1 P_{Z}^{\Phi}$; additional stress: $P_{Z}^{C}$ starting at $\mathrm{t}=5$. Damage levels equilibrate if the additional stress ends at $t_{1}=8.5$ minutes. If the stress lasts just half a minute more (ending at $t_{2}=9$ ), $D$ crosses the initial value-related tipping point (TP2), and the damage levels spiral out of control. Therefore, duration of pulsed studies matters, especially if multiple pulses are investigated: in case the time between pulses does not allow for a full recovery, the organism may experience a ratcheting mechanism: each cycle leaves more damage in the organism, eventually tipping the system. Parameter values listed in Table B.1 used, only $P_{Z}$ varied. 


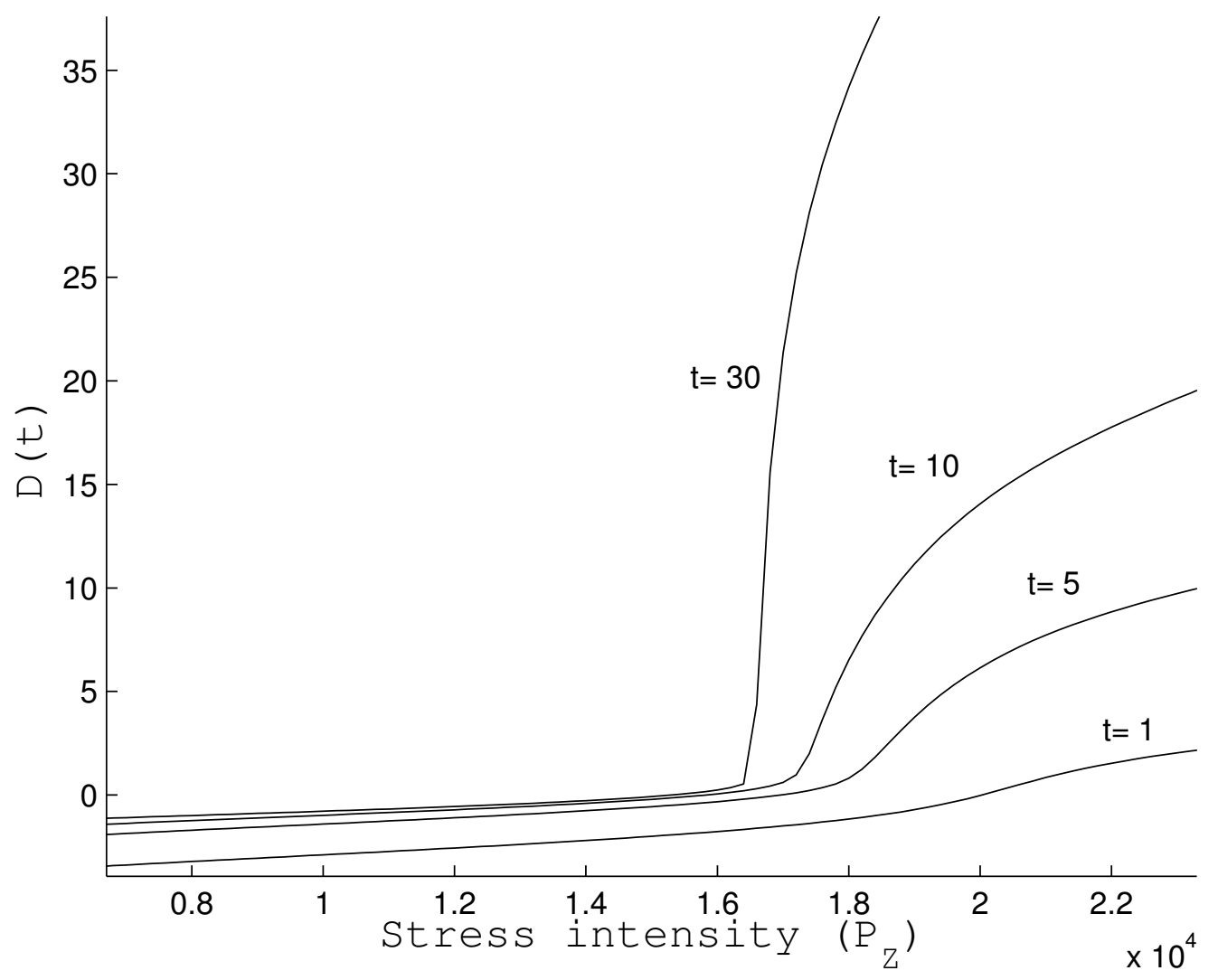

374

Figure 6: Transients affect perceived effects of stress depending on duration of observation, $t$. Damage at time $t$ (log scale) shown for a range of stressor intensity $\left(P_{Z}\right)$. Critical stress $\left(P_{Z}^{C}=\right.$ 16370) can be estimated by looking at the pattern of damage accumulation: once $P>P_{Z}^{C}$, damage accumulates indefinitely, thus if given enough time to accumulate resulting in a marked increase in accumulated damage (a 'kink' in the damage vs. exposure curve). Short observation periods do not leave enough time for ROS and damage to spiral out of control even when $P>P_{Z}^{C}$, so the transition (from slow to fast accumulation of damage) seems to happen for larger stress. As a result, $P_{Z}^{C}$ could be significantly overestimated; in this example, the estimate would be 22000 , 18000, and 17000 for $t=1,5$, and 10, respectively). A longer observation period ( $\mathrm{t}=30)$, however, correctly estimates $P_{Z}^{C} \approx 16400$. Parameter values listed in Table B.1 used, only $P_{Z}$ varied.

\section{Discussion}

We developed simple models for two generic types of indicators of cellular stress: proxies for damage (e.g. carbonylation, membrane permeability, electron transport function), and proxies for damage-inducing compounds (notably ROS). The models offer a minimal representation of 
currently accepted understanding of dominant processes in the cell to describe ROS creation due to metabolism and exposure to environmental stressors, regulation of ROS, damage inflicted by ROS and directly by environmental stressors, and regulation of damage. The complexity of cellular regulatory networks is condensed into simple negative feedback loops requiring one 'controller' for each controlled variable. The full model has four state variables (ROS, ROS control, damage, and damage control/repair), but model reduction is possible by recognizing that the damage dynamics are slow relative to other processes.

Although damage is the true "slow" variable, we focused much of our analysis on two state variables (ROS and damage) because these (or proxies) relate to quantities frequently measured in ecotoxicological studies. The analysis showed the possibility of: (i) tight regulation of ROS and damage in some conditions, and (ii) unbounded (runaway) increase of ROS and damage in others. The relationship between susceptibility and resistance to exposure defined by (11) inform about the conditions in which stable equilibrium can be achieved.

Runaway dynamics are a consequence of a positive feedback between ROS and damage resulting in uncontrolled increase in both: more ROS begets more damage, and more damage begets more ROS. Transitions from a stable, controlled state to runaway dynamics involve one of two types of tipping points:

- Type 1: parameter or forcing-induced tipping point. Type 1 tipping point can be reached by either bifurcation of a unique positive stable equilibrium (UPSE) into runaway dynamics (interpretable as a saddle node bifurcation at $D= \pm \infty$ or $D^{-1}=0$ ), or a saddle-node bifurcation as two equilibria approach and annihilate each other. The tipping point can be caused by changes in stressor intensity, but only if the effects of damage on ROS production are multiplicative or if the stressor directly affects ROS production or cell damage.

- Type 2: related mathematically to initial condition dependence or biologically to the history of the system under study: accumulation of damage and/or ROS above a critical level can cause a runaway of an otherwise controllable system. The transition can be induced by fluctuating stressors, for example a long and/or intense stress pulse, or by a ratchet-like 
response to periodic stress if damage from one cycle is not fully repaired before another cycle starts. This tipping point is of particular importance because it can occur in all investigated (non-linear) models whenever two steady states exist (see Table 2), and because it depicts long-term sensitivity to multiple and recurring stressors.

Our models were motivated by the large, and growing body of literature reporting simultaneously enhanced levels of ROS and of some measure of damage in response to environmental stress. Each of our models predicts that increase in direct stressor effects on ROS $\left(P_{Z}\right)$ or damage $\left(P_{D}\right)$ can cause long-term increase in both ROS and damage, irrespective of whether there are runaway dynamics or a stable steady state (Appendix D). Strong correlation between observed ROS and cellular damage may be caused by either direct contribution to ROS production, or direct damage production. Distinguishing between the two possibilities is possible in principle by comparing transients, but this requires time series rather than just endpoints. We know of no experimental study where this has been attempted. Note that lack of observable correlation between changes in ROS and cellular damage does not preclude direct stressor contribution to ROS production. Depending on the parameter values, the ratio of ROS and damage can increase, decrease, or remain constant with time and/or exposure (Appendix D). Also, the type of control mechanism might influence these results; although only one family of control mechanisms was considered here, others are possible and can be substituted in place of $R_{Z}$ and $R_{D}$ (e.g. integral control (Drengstig et al. 2012) or rein control (Saunders et al., 1998; Saunders et al., 2000), which could cause ROS to remain constant under stress).

Indeed, our analysis (Appendix C) highlights dynamic features that might be most susceptible to the choice of type of control. While all investigated types of control permit at least one steady state, details of the control determine the number and character of steady states. Interestingly, the analysis (Appendix C.2.2) shows that qualitative dynamics of a model with saturating damage control does not depend on whether ROS control is saturating or not. Since our analysis largely relied on generic properties common to many regulatory mechanisms (e.g. increase of regulation with stress, and possibility of overwhelming regulation), we expect that alternative functional 
forms based on the same consideration follow similar qualitative patterns. Choices of the details in any particular application are best informed by the prior knowledge of the modeled system, and experimental data.

The key to using our models to interpret experimental data is to have time resolved measurements of ROS and damage. Damage creation and repair are dynamical processes; even when ROS and control dynamics equilibrate rapidly, transients can still have significant consequences. For example, critical exposure can be overestimated due to transients (Figure 6); time-resolved measurements would show whether ROS and damage levels equilibrated, thus preventing masking of runaway dynamics by slow transients. That said, we appreciate the practical difficulty in obtaining the time resolved measurements, as the relevant procedures are commonly destructive.

Our model analyses used a time-scale separation argument to focus on the relatively slow dynamics of damage, so our conclusions only relate to the slow (long time scale) dynamics. Therefore, when modeling acute exposures, the validity of the time separation argument has to be scrutinized. The full four-dimensional model can exhibit very large transients in the 'fast dynamics' resulting from inclusion of all four state variables, but these are almost certainly biologically unrealistic as a wide range of mechanisms not included in the model may be implicated in ROS regulation over very short times. Therefore, a more detailed, context-specific characterization of ROS dynamics is necessary if aiming to model the fast dynamics.

Even in this minimal representation, all our models have at least one tipping point and could be used in conjunction with other process-based models, such as those of Dynamic Energy Budget (DEB) theory (see Leeuwen et al. 2010 for an example). With such combinations, ecologically relevant but experimentally elusive stressor impacts can be assessed. For example, a toxicokinetic model may require energy fluxes defined by a DEB model to properly account for effects of exposure. Since our models specify control and repair fluxes, the required energetic costs could be calculated and included in a DEB model to calculate sub-lethal stressor impacts such as those on growth and reproduction and, subsequently, on population dynamics.

The latter requires a model relating environmental stress to mortality, for which the concept 
of hazard in the DEB theory might be used. Hazard represents the instantaneous probability of dying caused by accumulation of irreversible damage, whose accumulation is essentially a mechanism of physiological aging. Adapting the model presented here to track irreversible damage is straightforward. Mathematically, it amounts to setting damage repair to zero $\left(g_{D}=0\right)$, but the interpretation of damage $(D)$ and damage yield $\left(y_{D}\right)$ subtly changes: only irreparably damaged cellular components are considered damage (e.g. irreparably damaged DNA, and reduced function of proteins produced by such DNA). The definition of yield changes accordingly to include only the production of newly created irreparable damage. Our model can track both repairable and irreparable damage using a different state variable for each type of damage, but special attention should be given to interaction between the two types of damage.

Although state variables were defined with distinct cellular processes in mind, simplification required a significant level of abstraction that comes with a cost in ability to relate state variables to measurable quantities. For example, damage produced by ROS is affected both by the species and the location of the ROS. The model framework set up here could easily be adapted to include some heterogeneity in ROS and account for various types of proxies for damage without affecting the qualitative dynamics leading to tipping points.

Although abstraction of state variables and cellular control processes creates challenges in applying the model to particular systems, it increases generality of the approach. All organisms have developed strategies to mitigate consequences of environmental stress and stress created by their own metabolism. These strategies more often than not rely on negative and positive feedbacks that impact the dynamics of damage and damage-inducing compounds. Therefore, with reinterpretation of the state variables, the model (or similar models) - as well as the concepts of susceptibility and resistance to exposure - becomes applicable to more complex, multicellular organisms. 


\section{Acknowledgements}

This work had its origins in many discussions with Gary Cherr, Hunter Lenihan, Robert Miller Patricia Holden, John Priester and other colleagues in the University of California Center for Environmental Implications of Nanotechnology. We also thank Jelena Repar for help with molecular biology. This material is based upon work supported by the National Science Foundation and the Environmental Protection Agency under Cooperative Agreement Number DBI-0830117. There was also partial support from (i) the US Environmental Protection Agency through STAR grant 835797, and (ii) Croatian National Science Foundation (HRZZ) project ACCTA grant 2202. Any opinions, findings, and conclusions or recommendations expressed in this material are those of the author(s) and do not necessarily reflect the views of the National Science Foundation, or the Environmental Protection Agency. This work has not been subjected to EPA review and no official endorsement should be inferred.

\section{REFERENCES}

Bannister J.V., Bannister W.H., Rotilio G. 1987. Aspects of the structure, function, and applications of superoxide dismutase. CRC Critical Reviews in Biochemistry 22 (2): 111-80.

Chondrogianni, N., Isabelle Petropoulos, Stefanie Grimm, Konstantina Georgila, Betul Catalgol, Bertrand Friguet, Tilman Grune, Efstathios S. Gonos. 2014. Protein damage, repair and proteolysis. Molecular Aspects of Medicine 35:1-71

Culotta, V.C. and Daly, M.J. (2013) Manganese Complexes: Diverse Metabolic Routes to Oxidative Stress Resistance in Prokaryotes and Yeast. Antioxidants \& Redox Signaling, 19(9), 933-944.

Drengstig, T., Jolma, I.W., Ni, X.Y., Thorsen, K., Xu, X.M., and Ruoff, P. 2012. A Basic Set of Homeostatic Controller Motifs. Biophysical Journal 103(9):2000-2010

Friedman N., Vardi S., Ronen M., Alon U. and Stavans, J. 2005 Precise temporal modulation in the response of the SOS DNA repair network in individual bacteria. PLoS Biol 3(7):e238 
Giorgio, M., Trinei, M., Migliaccio, E. and Pelicci, P.G.. 2007. Hydrogen peroxide: a metabolic by-product or a common mediator of ageing signals? Nature reviews: molecular cell biology 8(9):722-728.

Gros, L., Murat K Saparbaev and Jacques Laval 2002. Enzymology of the repair of free radicals-induced DNA damage. Oncogene 21:8905-8925.

Imlay, J.A. 2003. Pathways of Oxidative Damage. Annu. Rev. Microbiol. 57:395-418.

Ivask, A., ElBadawy, A., Kaweeteerawat, C., Boren, D., Fischer, H., Ji, Z., Chang, C.H., Liu, R., Tolaymat, T., Telesca, D., Zink, J.I., Cohen, Y., Holden, P.A. and Godwin, H.A. 2014. Toxicity Mechanisms in Escherichia coli Vary for Silver Nanoparticles and Differ from Ionic Silver. ACS Nano 8(1):374-386. DOI:10.1021/nn4044047

Jager, T. and Zimmer E.I. 2012. Simplified Dynamic Energy Budget model for analysing ecotoxicity data. Ecological Modelling 225:74-81 DOI:10.1016/j.ecolmodel.2011.11.012

Jager, T., Barsi A., Hamda, N.T., Martin, B.T., Zimmer, E.I. and Ducrot, V. 2014a. Dynamic energy budgets in population ecotoxicology: applications and outlook. Ecological Modelling 280:140-147 DOI:10.1016/j.ecolmodel.2013.06.024

Jager, T., Gudmundsdóttir, E.M. and Cedergreen, N. 2014b. Dynamic modeling of sub-lethal mixture toxicity in the nematode Caenorhabditis elegans. Environ Sci Technol 48:7026-7033 DOI:10.1021/es501306t

Jamet, A., Sigaud, S. Van de Sype, G., Puppo, A. and Hérouart, D. 2003. Expression of the Bacterial Catalase Genes During Sinorhizobium meliloti-Medicago sativa Symbiosis and Their Crucial Role During the Infection Process. The American Phytopathological Society 16(3):217-225.

Kaweeteerawat, C., Chang, C.H., Roy, K.R., Liu, R., Li, R., Toso, D., Fischer, H., Ivask, A., Ji, Z., Zink, J.I., Zhou, Z.H., Chanfreau, G.F., Telesca, D., Cohen, Y., Holden, P.A., Nel, A.E. and Godwin, H.A. 2015. Cu Nanoparticles Have Different Impacts in Escherichia coli and Lactobacillus brevis than Their Microsized and Ionic Analogues. ACS Nano 9(7):7215-7225. DOI: $10.1021 /$ acsnano.5b02021

Klanjscek, T., Nisbet, R.M., Priester, J.H., Holden, P.A. 2012. Modeling physiological pro- 
571 572

cesses that relate toxicant exposure and bacterial population dynamics. PLoS ONE 7(2):e26955 DOI:10.1371/journal.pone.0026955

Klanjscek, T., Nisbet, R.M., Priester, J.H. and Holden, P.A. 2013. Dynamic energy budget approach to modeling mechanisms of CdSe quantum dot toxicity. Ecotoxicology 22:319-330 DOI:10.1007/s10646-012-1028-7

Kooijman, S.A.L.M. 2010. Dynamic Energy Budget Theory for Metabolic Organisation, 3rd ed. Cambridge University Press, Great Britain. ISBN 9780521131919.

van Leeuwen, I.M., Vera, M.J. and Wolkenhauer, O.. 2010. Dynamic energy budget approaches for modelling organismal ageing. Phil. Trans. R. Soc. B 365:3443-3454

Martin, B., Jager, T., Nisbet, R.M., Preuss, T.G. and Grimm V. 2013. Predicting population dynamics from the properties of individuals: a cross-level test of Dynamic Energy Budget theory. American Naturalist 181(4):506-519 DOI: 10.1086/669904

Nel, A., Xia, T., Mädler, L. and Li, N. 2006. Toxic potential of materials at the nanolevel Science 311(5761): 622-7.

Priester, J.H., Stoimenov, P.K., Mielke, R.E., Webb, S.M., Ehrhardt, C., Zhang, J.P., Stucky, G.D. and Holden, P.A. 2009. Effects of soluble cadmium salts versus CdSe quantum dots on the growth of planktonic Pseudomonas aeruginosa. Environmental Science \& Technology 43(7):2589-2594.

DOI:10.1021/es802806n

Priester, J.H., Ge, Y., Mielke, R.E., Horst, A.M., Cole-Moritz, S., Espinosa, K., Gelb, J., Walker, S.L., Nisbet, R.M., An, Y.J., Schimel, J.P., Palmer, R.G.; Hernandez-Viezcas, J.A.; Zhao, L., Gardea-Torresdey, J. L. and Holden, P.A. 2012. Soybean susceptibility to manufactured nanomaterials with evidence for food quality and soil fertility interruption. Proceedings of the National Academy of Sciences of the United States of America 109(37):E2451-E2456.

Saunders, P.T., Koeslag, J.H. and Wessels, J.A. 1998. Integral rein control in physiology. Journal of Theoretical Biology 194(2):163-173

Saunders, P.T., Koeslag, J.H. and Wessels, J.A. 2000. Integral rein control in physiology II: a general model. Journal of Theoretical Biology 206(2):211-220 
Zahradka, K., Slade, D., Bailone, A., Sommer, S., Averbeck, D., Petranovic, M., Lindner, A.B. and Radman, M. 2006. Reassembly of shattered chromosomes in Deinococcus radiodurans. Nature $443,569-573$

Zhang, Q. and Andersen, M.E. 2007. Dose Response Relationship in Anti-Stress Gene Regulatory Networks. PLoS Computational Biology 3(3):e24 DOI:10.1371/journal.pcbi.0030024

\section{APPENDICES}

\section{A Dynamics of controller compounds}

In our model, ROS inactivation and damage repair function as controls of ROS and damage. The control is mediated by controller compounds (controllers) that are produced by the cell, interact with the controlled compounds (inactivate ROS and/or repair damage), and decay (see Table A.1). We considered two types of mechanisms for the control, depending on the type of interaction between controllers and ROS/damage: linear and saturating. The ROS controller dynamics and control of ROS is detailed below; the rationale of damage control is similar.

Table A.1: ROS and damage dynamics, complete with the dynamics of their respective controllers, $E$ and $A$.

\begin{tabular}{|c|c|c|}
\hline dynamics & fluxes & \\
\hline \multirow{3}{*}{$\frac{d Z}{d t}=j_{Z, p r o d}-k_{Z} Z-j_{Z E}$} & $j_{Z, p r o d}$ & ROS production due to metabolism and stress \\
\hline & $k_{Z} Z$ & spontaneous ROS reaction flux (causing damage) \\
\hline & $J Z E$ & controlled ROS removal flux (mediated by $E$ ) \\
\hline \multirow{2}{*}{$\frac{d E}{d t}=j_{E, \text { prod }}-j_{E, \text { out }}$} & $j_{E, p r o d}$ & saturating production flux of $E$ in response to ROS \\
\hline & $j_{E, \text { out }}$ & inactivation flux of the controller compound $E$ \\
\hline \multirow{2}{*}{$\frac{d A}{d t}=j_{A, \text { prod }}-j_{A, \text { out }}$} & $j_{A, p r o d}$ & saturating production flux of $A$ in response to damage \\
\hline & $j_{A, \text { out }}$ & inactivation flux of the controller compound $A$ \\
\hline \multirow{2}{*}{$\frac{d D}{d t}=j_{D, p r o d}-j_{D A}$} & $j_{D, p r o d}$ & damage production due to spontaneous ROS reactions \\
\hline & $j_{D A}$ & damage repair (mediated by $A$ ) \\
\hline
\end{tabular}

We assume that the production of $E$, the ROS controller, is induced in the presence of ROS. Therefore, the production of $E$ increases as ROS levels increase but, recognizing that the upregu- 
lation potential of a cell is finite, we assume a Michaelis-Menten type of saturating dynamics:

$$
j_{E, p r o d}=\frac{v_{E} Z}{K_{E}+Z}
$$

where $v_{E}$ is the maximum production rate of $E$, and $K_{E}$ is the induction saturation constant.

Controller compounds are inactivated due to cellular turnover, interactions with controlled compounds, and/or targeted degradation mechanisms. We choose simple, linear dynamics:

$$
j_{E, \text { out }}=k_{E} E
$$

where $k_{E}$ is the controller inactivation rate.

In the case of linear control of ROS inactivation, we assumed that controlled ROS inactivation depends on the contact rate between the controller, $E$, and ROS, $Z$, resulting in a simple bilinear collision term:

$$
j_{Z E}^{\text {linear }}=g_{Z} E Z
$$

with $g_{Z}$ signifying the ROS control strength coefficient.

For the saturating control of ROS inactivation, we considered that there may be an appreciable 'handling time' for inactivation of ROS by $E$ and, consequently, assumed that:

$$
j_{Z E}^{\text {saturating }}=g_{Z} E Z \frac{K_{Z}}{K_{Z}+Z}
$$

where $K_{Z}$ is the relative saturation coefficient.

If stress intensities change slowly relative to the dynamics of controllers, e.g. because the bioaccumulation of toxic stressors is gradual, and if stress intensities do not exceed a value that would cause rapid unbounded increase in ROS and damage, we can set $d E / d t=0$ and solve the corresponding equation in Table A.1 for a quasi-equilibrium value of $E$ :

$$
E^{*}=\frac{v_{E}}{k_{E}} \frac{Z}{K_{E}+Z}
$$


607 Inserting A.5 into the equation for $d Z / d t$ in Table A.1 gives

$$
R_{Z}=\left\{\begin{array}{ll}
\frac{v_{Z} Z^{2}}{K_{E}+Z} & \text { for } j_{Z E}^{\text {linear }} \\
\frac{v_{Z} Z^{2}}{\left(K_{E}+Z\right)\left(1+Z / K_{Z}\right)} & \text { for } j_{Z E}^{\text {saturating }}
\end{array},\right.
$$

608 where $v_{Z}=g_{Z} v_{E} / k_{E}$. Equivalently, with $v_{D}=g_{D} v_{A} / k_{A}$,

$$
R_{D}= \begin{cases}\frac{v_{D} D^{2}}{K_{A}+D} & \text { for } j_{D A}^{\text {linear }} \\ \frac{v_{D} D^{2}}{\left(K_{A}+D\right)\left(1+D / K_{D}\right)} & \text { for } j_{D A}^{\text {saturating }}\end{cases}
$$




\section{B Parameter values}

610

611

612

Table B.1: Symbols, state variables, parameters, and reference values. The reference values were used in simulations unless otherwise noted. The acronym "u.of" stands for "units of".

\begin{tabular}{|c|c|c|}
\hline symbol & meaning & reference values \\
\hline \multicolumn{3}{|c|}{ State variables and flux symbols } \\
\hline$Z$ & damage-inducing compounds (ROS) & - \\
\hline$D$ & cellular damage & - \\
\hline$E$ & controller variable - ROS control & - \\
\hline$A$ & controller variable - damage repair & - \\
\hline$S$ & stressor intensity & - \\
\hline$P_{0}$ & metabolic ROS production flux & - \\
\hline$P_{Z}$ & stress-related ROS production flux & - \\
\hline$P_{D}$ & stress-related damage production flux & - \\
\hline$R_{Z}$ & regulated ROS clearance & - \\
\hline$R_{D}$ & regulated damage repair & - \\
\hline \multicolumn{3}{|c|}{ parameters (alphabetical order) } \\
\hline$g_{D}$ & damage repair coefficient & 0.001 minute $^{-1}$ \\
\hline$g_{Z}$ & ROS control strength coefficient & 25 minute $^{-1}$ \\
\hline$g_{Z D}$ & multiplicative damage interaction coeff. & $0.1(\text { u.of D })^{-1}$ \\
\hline$\gamma_{D S}$ & stress-related damage production coeff. & 0 \\
\hline$\gamma_{Z D}$ & additive damage interaction coefficient & 0 \\
\hline$\gamma_{Z S}$ & stress-related ROS production coefficient & included in $P_{Z}$ \\
\hline$K_{A}$ & half-saturation constant for production of $A$ & 1 u.of $\mathrm{A}$ \\
\hline$K_{D}$ & relative saturation coefficient of damage repair & 1 u.of D \\
\hline$K_{E}$ & half-saturation constant for production of $E$ & 1 u.of $\mathrm{E}$ \\
\hline$K_{Z}$ & relative saturation coefficient of ROS control & 1 u.of $\mathrm{Z}$ \\
\hline$k_{A}$ & clearance/turnover rate of $A$ & 0.012 minute $^{-1}$ \\
\hline$k_{E}$ & clearance/turnover rate of $E$ & 0.012 minute $^{-1}$ \\
\hline$k_{Z}$ & passive ROS clearance rate coefficient & 50 minute $^{-1}$ \\
\hline$v_{A}$ & maximum production rate of $A$ & 5 u.of $\mathrm{A} / \mathrm{minute}$ \\
\hline$v_{D}$ & damage control rate coefficient & $g_{D} v_{A} / k_{A}$ \\
\hline$v_{E}$ & maximum production rate of $E$ & 5 u.of $\mathrm{E} /$ minute \\
\hline$v_{Z}$ & ROS control rate coefficient & $g_{Z} v_{E} / k_{E}$ \\
\hline$y_{D}$ & damage yield from passive ROS reactions & 0.001 \\
\hline
\end{tabular}

Although we aimed at characterizing the dynamics of our model variants qualitatively, we used plausible parameter values whenever possible. All parameter values, and state variables are listed in Table B.1.

Here we show our reasoning for choosing particular values for clearance rates $\left(k_{Z}, k_{E}\right.$, and $\left.k_{A}\right)$, 
and maximum production/collision rates: $\left(v_{E}, v_{A}, g_{Z}\right.$, and $\left.g_{D}\right)$. For this purpose, we take the SOS DNA repair network in Escherichia coli as a paradigm for our system and assume that the dynamics of the RecA protein of this repair network, as quantified by Friedman et al. (2005), are representative for those of the controller compounds in our system.

Friedman et al. (2005) used UV radiation as a stressor to induce the promoter activity of a fusion product of the RecA promoter and a fluorescent protein. At saturating levels of UV radiation, the expression rate of this fusion product is about 5 arbitrary unites (au) per minute (see Figure 1C in Friedman). Accordingly, we take $v_{E}=v_{A}=5 \mathrm{au} / \mathrm{min}$.

Nath and Koch (1971) estimated the half-life of proteins in starved E. coli at about 60 minutes. Assuming a similar half-life for the controllers in our system,

$$
k_{A}=k_{E}=\frac{\ln (2)}{60}=0.012 \text { per minute. }
$$

This rate is consistent with RecA decay measured after a peak in expression in Deinococcus radiodurans (Vlašić et al. 2008).

Panel F from Friedman's et al. (2005) Figure 1 shows that - for low exposures - promoter activity starts to decline within 35 minutes, consistent with the estimate of 40 minutes for the repair response of the SOS gene cascade (Michel 2005). If the decline in promoter activity results from successful damage repair (i.e. no need for the expression of repair mechanism proteins once the damage is repaired), we can use the time of decline as a proxy for time to successful repair. Assuming double the exposure creates double the damage, we can then use panels $\mathrm{D}$ and $\mathrm{G}$ to estimate the repair rate at maximum expression. Following a $20 \mathrm{~J} / \mathrm{m}^{2}$ exposure (Panel D), it takes about 70 minutes for the reduction in promoter activity to start; following a $50 \mathrm{~J} / \mathrm{m}^{2}$ exposure, the time to reduction is approximately 130 minutes. Hence, the half-life of damage is about 60 minutes * when regulation is at the maximum*, i.e. when 


$$
\begin{gathered}
A_{\max }=\quad \frac{v_{A}}{k_{A}} \approx 420, \text { and } \\
g_{D}=\frac{\ln (2)}{\tau_{0.5} A_{\max }}=\frac{0.70}{60 \cdot 500}=3 \cdot 10^{-5} .
\end{gathered}
$$

639

640

Such a low value of made the equilibrium hard to attain, so we used a larger value of 0.001 per minute. The ROS clearance rate $\left(k_{Z}\right)$ and interaction coefficient $\left(g_{Z}\right)$ should be significantly higher because ROS dynamics is extremely fast; we arbitrarily set them to 25 per minute and 50 per minute, respectively. The following values were used for other parameters: yield coefficient $y_{D}=0.001$, strength of effect of damage on ROS production $g_{Z D}=0.1$. All saturation constants were set to unity.

\section{B.1 REFERENCES}

Friedman N., Vardi S., Ronen M., Alon U. and Stavans, J. 2005. Precise temporal modulation in the response of the SOS DNA repair network in individual bacteria. PLoS Biol 3(7):e238

Nath, K. and Koch, A.L. 1971. Chemistry and Metabolism of Macromolecules: Protein Degradation in Escherichia coli : II. Strain Differences in the Degradation of Protein and Nucleic Acid Resulting from Starvation. J. Biol. Chem. 246:6956-6967

Michel, B. 2005. After 30 years of study, the bacterial SOS response still surprises us. PLoS Biol 3(7):e255.

Vlašić, I., Ivančić-Baće, I., Imešek, M., Mihaljević, B. and Brčić-Kostić, K. 2008. RecJ nuclease is required for SOS induction after introduction of a double-strand break in a RecA loading deficient recB mutant of Escherichia coli. Biochimie 90:1347-1355. 


\section{${ }_{656}$ C Equilibrium states and stability}

${ }_{657}$ The models in Table 2 can be written in a general form:

$$
\begin{array}{ccc}
\frac{d Z}{d t}= & P_{Z}+D\left(\gamma_{Z D}+g_{Z D} P_{Z}\right)-k_{Z} Z-R_{Z} \\
\frac{d D}{d t}= & y_{D} k_{Z} Z+P_{D}-R_{D}
\end{array}
$$

658 659 negative and the determinant is positive, i.e.

$$
\Phi_{Z Z}^{-}+\Phi_{D D}^{-}<0, \text { and } \Phi_{Z Z}^{-} \Phi_{D D}^{-}-\Phi_{Z D}^{+} \Phi_{D Z}^{+}>0
$$

667 when evaluated at $\left(Z^{*}, D^{*}\right)$. Since the first inequality is always satisfied, the stability is determined 668 by the second inequality, which has a simple graphical interpretation in the Z-D plane. First, let

Since each of the functions $R_{Z}$ and $R_{D}$ in Table (1) is a monotonic increasing function of its argument, the respective partial derivatives are positive, and both $\Phi_{Z Z}^{-}$and $\Phi_{D D}^{-}$are negative for any combination of parameters (with $k_{Z}, v_{Z}, v_{D}>0$ ), i.e. $\Phi_{Z Z}^{-}+\Phi_{D D}^{-}<0$. From the Bendixson criterion (McCluskey and Muldowney 1998), there can therefore never be periodic solutions of the equations. Thus, the only possible attractors are fixed points (equilibria).

An equilibrium $\left(Z^{*}, D^{*}\right)$ is stable if the trace of the Jacobian evaluated at the equilibrium is 
669

us consider the two isoclines:

$$
\begin{aligned}
D(Z) & =\frac{1}{g_{Z D} P_{Z}+\gamma_{Z D}}\left(k_{Z} Z+R_{Z}-P_{Z}\right), \text { the "Z-isocline" (in Z-D space) } \\
\text { and } Z(D) & =\frac{1}{y_{D} k_{Z}}\left(R_{D}-P_{D}\right), \text { the "D-isocline" (in D-Z space), }
\end{aligned}
$$

670 whose slopes (in Z-D plane) are

$$
\begin{gathered}
a_{Z}=\frac{\partial D(Z)}{\partial Z}=\frac{k_{Z}+\partial R_{Z} / \partial Z}{g_{Z D} P_{Z}+\gamma_{Z D}}=\frac{-\Phi_{Z Z}^{-}}{\Phi_{Z D}^{+}} \\
a_{D}^{-1}=\frac{\partial Z(D)}{\partial D}=\frac{\partial R_{D} / \partial Z}{y_{D} k_{Z}}=\frac{-\Phi_{D D}^{-}}{\Phi_{D Z}^{+}}
\end{gathered}
$$

671 where $a_{D}$ in Z-D plane is an inverse of the slope calculated in D-Z plane. Reorganizing the second 672 inequality in (C.5), while taking the known signs from (C.4) into the account, gives:

$$
\begin{aligned}
\frac{\left|\Phi_{Z Z}^{-}\right|}{\left|\Phi_{Z D}^{+}\right|} & >\frac{\left|\Phi_{D Z}^{+}\right|}{\left|\Phi_{D D}^{-}\right|} \\
a_{Z} & >a_{D},
\end{aligned}
$$

673 i.e. when evaluated in the Z-D plane at the equilibrium, the slope of the Z-isocline must be greater 674 than the slope of the D-isocline.

675 676

Note that $R_{Z}(0)=R_{D}(0)=0$, implying that for any $P_{Z}>0$ and $P_{D} \geq 0, D(0)<0$ and $Z(0) \leq 0$. Hence, the intercepts of the $\mathrm{D}$-isocline on the $\mathrm{D}$ axis and of the $\mathrm{Z}$-isocline on the $\mathrm{Z}$ axis are both positive. Furthermore, if (C.11) holds in the limit as $Z, D \rightarrow \infty$, then for sufficiently large values of $Z$, the value of $D$ on the $Z$-isocline always exceeds its value on the $\mathrm{D}$-isocline. Since the opposite holds when $Z=0$, there must be at least one intersection. Thus, a sufficient condition for the existence of at least one steady state is

$$
\lim _{D, Z \rightarrow \infty}\left(\Phi_{Z Z}^{-} \Phi_{D D}^{-}-\Phi_{Z D}^{+} \Phi_{D Z}^{+}\right)>0
$$

${ }_{681}$ i.e. equation (11) in the text. To determine the number of possible equilibria, we continue to use 
682

683

684

685

686

687

the isoclines in the positive quadrant of the Z-D plane, but further analysis requires specification of $R_{Z}$ and $R_{D}$.

\section{C.1 Linear interaction terms}

When the interaction terms defining ROS inactivation and damage repair are linear in $\mathrm{Z}$ and $\mathrm{D}$ respectively, the functions $R_{Z}$ and $R_{D}$ from Table 1 are

$$
R_{Z}=\frac{v_{Z} Z^{2}}{K_{E}+Z} ; \quad R_{D}=\frac{v_{D} D^{2}}{K_{A}+D}
$$

Starting with the Z-isocline in the Z-D plane

$$
\frac{\partial^{2} D(Z)}{\partial Z^{2}} \propto \frac{\partial^{2} R_{Z}}{\partial Z^{2}}>0
$$

thus the Z-isocline is concave upward (convex) in the Z-D plane. Similarly, the D-isocline is concave upward in the D-Z plane:

$$
\frac{\partial^{2} Z(D)}{\partial D^{2}} \propto \frac{\partial^{2} R_{D}}{\partial D^{2}}>0,
$$

i.e. concave downward in the Z-D plane. Since i) both isoclines increase with Z (equations (C.8) and (C.9)), ii) the Z-isocline is concave upward while the D-isocline is concave downward, and iii) the Z-isocline has a positive intercept on the D-axis, and the D-isocline has a positive intercept on the Z-axis, the isoclines can only intersect once in the positive quadrant of the Z-D plane. Therefore, at most one (unique) positive steady state exists, provided that for sufficiently large $Z$ inequality (C.11) is satisfied, i.e. $a_{Z}>a_{D}$. This unique steady state also must be stable because the concavity of both isoclines remains the same, so if (C.11) is true for any $Z>0$, the UPSE condition (11) is also necessarily true. Note that the proof of this result relies only on the concavity of the isoclines, not on our specific choice of functional forms (C.13). 


\section{C.2 Saturating interaction terms}

In this section, we investigate steady states and stability of models with one or both saturating terms. First, we investigate the double-saturating model (saturating control of both ROS and damage). In addition to the equations above, we will need:

1. first derivatives of the isoclines with respect to the independent variable:

$$
\begin{aligned}
g_{Z D} \frac{\partial D}{\partial Z} & =k_{Z}+\frac{v_{Z} Z\left(Z\left(K_{E}+K_{Z}^{-1}\right)+2 K_{E} K_{Z}^{-1}\right)}{\left(K_{E}+Z\right)^{2}\left(K_{Z}^{-1}+Z\right)^{2}}>0 \text { for } \mathrm{Z}>0 . \\
y_{D} k_{Z} \frac{\partial Z}{\partial D} & =\frac{v_{D} D\left(D\left(K_{A}+K_{D}^{-1}\right)+2 K_{A} K_{D}^{-1}\right)}{\left(K_{A}+D\right)^{2}\left(K_{D}^{-1}+D\right)^{2}}>0 \text { for } \mathrm{D}>0
\end{aligned}
$$

2. second derivatives of the isoclines with respect to the independent variable:

$$
\begin{aligned}
g_{Z D} \frac{\partial^{2} D}{\partial Z^{2}} & =\frac{2 v_{Z}\left(2 K_{E}^{2} K_{Z}^{-2}-3 K_{Z}^{-1} K_{E} Z^{2}-\left(K_{E}+K_{Z}^{-1}\right) Z^{3}\right)}{\left(K_{E}+Z\right)^{3}\left(K_{Z}^{-1}+Z\right)^{3}} \\
y_{D} k_{Z} \frac{\partial^{2} Z}{\partial D^{2}} & =\frac{2 v_{D}\left(2 K_{A}^{2} K_{D}^{-2}-3 K_{D}^{-1} K_{A} D^{2}-\left(K_{A}+K_{D}^{-1}\right) D^{3}\right)}{\left(K_{A}+D\right)^{3}\left(K_{D}+D\right)^{3}}
\end{aligned}
$$

3. more convenient definitions of the isoclines:

(a) we re-define the Z-isocline (C.6) as $D_{1}(Z)=D(Z)$, and

(b) since the D-isocline in D-Z plane (C.7) is monotonic and therefore has an inverse, we can define it in Z-D plane as $D_{2}(Z)$.

During the analysis, we utilize the following statements regarding the Z-isocline $\left(Z_{I S O}\right)$ and D-isocline $\left(D_{I S O}\right)$ :

1. Except in a singular case of a touching intersection, models with saturating damage repair can only have an even number of steady states $(0,2,4 \ldots)$ for $Z, D>0$. Proof: for $Z=0$, $D_{1}(Z)<D_{2}(Z)$. Furthermore, note that $D_{2}$ diverges at $Z_{C}=\lim _{D \rightarrow \infty} Z(D)$, so the domain of $D_{2}$ in the first quadrant is $\left[0, Z_{C}\right]$. Also, $\exists \epsilon_{C}>0 \mid D_{1}\left(Z_{C}-\epsilon\right)<D_{2}\left(Z_{C}-\epsilon\right) \forall \epsilon<\epsilon_{C}$. Hence, 
on the both ends of the domain $\left(0, Z_{C)}\right), D_{1}(Z)<D_{2}(Z)$. This is only possible if isoclines intersect an even number of times, or if $D_{1}(Z) \leq D_{2}(Z) \forall Z \in\left[0, Z_{C}\right]$. The latter is satisfied when there are no intersections, or in the singular case of a touching intersection $\left(a_{Z}=a_{D}\right.$ at the intersection).

2. The positive steady state at (for $Z_{1}^{*}$ ) closest to the origin is stable. Proof: since $D_{1}(Z)<$ $D_{2}(Z) \forall Z \in\left[0, Z_{1}^{*}\right), d D_{1} /\left.d Z\right|_{Z_{1}^{*}}>d D_{2} /\left.d Z\right|_{Z_{1}^{*}}$. Therefore, unless the slopes are exactly equal, the condition (C.11) is satisfied, and the first equilibrium in the first quadrant (i.e. the equilibrium for the smallest $Z>0$ ) is stable.

3. In the first quadrant $(Z, D>0)$, the second derivatives of the isoclines are decreasing functions of their respective independent variables. This is readily discernible from equations (C.18) and (C.19): the greater the independent variable, the smaller the nominator, and the larger the denominator. Note that this means that $d^{2} D_{1} / d Z^{2}$ can only decrease, and that $d^{2} D_{2} / d Z^{2}$ can only increase.

4. Isoclines with saturating control can have only one inflection point. Proof: since, when evaluated at 0 , both second derivatives ((C.18) and (C.19)) are greater than zero, statement 3 guarantees that, as $Z$ and $D$ increase, (C.18) and (C.19) can intersect the x-axis only once. Hence, the second derivatives have only one positive root and, therefore, the isoclines have only one inflection point.

\section{C.2.1 Model with saturating control of both ROS and damage}

We start the analysis by noting that for $Z=0, D_{1}<D_{2}$ and $0=d D_{1} / d Z<d D_{2} / d Z$. For the isoclines to intersect, $d D_{1} / d Z$ has to increase, and $d D_{2} / d Z$ has to decrease. Since (at $\left.Z=0\right) d^{2} D_{1} / d Z^{2}>0$ and $d^{2} D_{2} / d Z^{2}<0$, the intersection is possible. As $Z$ increases, $d^{2} D_{1} / d Z^{2}$ decreases, while $d^{2} D_{2} / d Z^{2}$ increases until at least one isocline reaches the inflection point (second derivative equal to zero). If the isoclines intersect once, the second intersection (at $Z_{2}^{*}$ ) is only possible if at least one of the isoclines switches concavity for $Z<Z_{2}^{*}$ (otherwise the isoclines would continue to diverge). 
Note that, at the second intersection, $d D_{1} /\left.d Z\right|_{Z_{2}^{*}}<d D_{2} /\left.d Z\right|_{Z_{2}^{*}}$. For the third intersection to exist, the reverse would have to be true; hence, either $d D_{1} / d Z$ would have to increase, and/or $d D_{2} / d Z$ would have to decrease.

Depending on which isocline(s) reached the inflection point, we distinguish three possibilities:

- P1: Only $D_{2}$ reached the inflection point: both isoclines are concave upwards $\left(d^{2} D_{1} / d Z^{2}, d^{2} D_{2} / d Z^{2}>0\right)$. Since $D_{2}$ has to remain concave upwards (statement 4 ), $d D_{2} / d Z$ can only increase further. Hence, only a sufficient increase in $d D_{1} / d Z$ could cause a third intersection. Such an increase is, however, impossible (statement 3).

- P2: Only $D_{1}$ reached the inflection point: both isoclines are concave downwards $\left(d^{2} D_{1} / d Z^{2}, d^{2} D_{2} / d Z^{2}<0\right)$. Since $D_{1}$ has to remain concave downwards (statement 4 ), only a sufficient decrease in $d D_{2} / d Z$ could cause a third intersection; such an increase is impossible (statement 3).

- P3: Both isoclines reached the inflection point, so $D_{1}$ is concave downwards and $D_{2}$ is concave upwards. Since there are no additional inflection points, the isoclines will continue to diverge, making a third intersection impossible.

Therefore, the third intersection is impossible, i.e. there is a maximum of two intersections. Statement 1 then guarantees that (baring the singular case), there are either zero or two intersections; if two states exist, the one closer to the origin is stable (statement 2).

\section{C.2.2 Model with linear control of ROS and saturating control of damage}

Here, only $D_{2}$ can have an inflection point; $D_{1}$ remains concave upwards. However, all statements and steps in the analysis of the double-saturating model apply, with the exception that P2 and P3 need not be considered. Therefore, the results are the same: ignoring the singular case, this model can have either zero or two steady states, and the steady state closest to the origin is stable.

Note that models with saturating control of damage have a similar qualitative dynamics (in terms of existence of steady states) regardless of whether ROS control saturates or not. This is because, even when ROS control is saturated, ROS continues to be inactivated in the process of 


\section{${ }_{784}$ D Correlations between ROS and damage} ROS and damage.

\section{C.2.3 Model with saturating control of ROS and linear control of damage} and condition (11) needs to be considered. Two cases are possible: (11) is satisfied. stable.

\section{C.3 REFERENCES} Review 40(4):931-934 $Z$ and $D$ as forcing $\left(P_{Z}\right.$ or $\left.P_{D}\right)$ increases, and 2$)$ the ratio of $Z$ and $D$ in a runaway.

damage creation: mathematically, the inactivation $(\sim Z)$ is (in terms of dynamics) indistinguishable from linear control for large $Z\left(\lim _{Z \rightarrow \infty} R_{Z} \sim Z\right)$. Hence, even when the ROS control is saturated for large $Z$, the inactivation term assumes the dynamical role equivalent to that of linear ROS control. Clearly, the actual dynamics will differ due to the positive feedback loop between

Here, $D_{1}$ can have an inflection point, but $D_{2}$ cannot. Therefore, statement 1 is not applicable,

1. Condition (11) is satisfied. Then, $D_{1}<D_{2}$ for small and $D_{1}>D_{2}$ for large values of $Z$. This is only possible if there is an odd number of intersections $(1,3,5 \ldots)$. Since more than two intersection are not possible, there is one and only one intersection whenever condition

2. Condition (11) is not satisfied. Then, $D_{1}<D_{2}$ for both small and large values of $Z$. Therefore (ignoring the singular case and recognizing that the third steady state is impossible), either zero or two intersections exist. Statement 2 holds, so the steady state closer to the origin is

McCluskey, C.C. and Muldowney, J.S. 1998. Stability Implications of Bendixson's Criterion. SIAM

Here we look at how $Z$ changes relative to $D$ by a qualitative analysis of 1 ) equilibrium values of 


\section{D.1 Changes in the equilibrium as forcing increases}

Let $a=\partial D(Z) /\left.\partial Z\right|_{*}$ be the rate of change of $Z$-isocline (C.6) with respect to $Z$, and $b=\partial Z(D) /\left.\partial(D)\right|_{*}$ the rate of change of $D$ isocline (C.7) with respect to $D$ at the stable equilibrium. For the equilibrium to exist, the two isoclines need to intersect, and for the equilibrium to be stable, the $Z$-isocline has to be steeper than the $D$-isocline $\left(a>b^{-1}\right)$.

We start by linearizing the isoclines at the steady state, $Z^{*}$ and $D^{*}$, and consider an infinitesimal increase in $P_{Z}$ resulting in changes of the equilibrium, $\delta Z^{*}$ and $\delta D^{*}$. Since $P_{Z}$ affects only the $Z$ isocline, it is appropriate to observe the shift of the equilibrium along the $D$ isocline,

$$
\delta Z^{*}=b \delta D^{*} .
$$

The ratio of $\delta D^{*}$ and $\delta Z^{*}$ is, therefore, equal to $b^{-1}$ :

$$
b^{-1}=\left(\frac{\partial Z(D)}{\partial D}\right)_{*}^{-1}=y_{D} k_{Z}\left(\frac{\partial R_{D}}{\partial D}\right)_{*}^{-1}=y_{D} k_{Z}\left\{\begin{array}{ll}
\frac{\left(K_{A}+D^{*}\right)^{2}}{D^{*} v_{D}\left(D^{*}+2 K_{A}\right)} & \text { for } j_{D A}^{\text {linear }} \\
\frac{\left(K_{A}+D^{*}\right)^{2}\left(K_{D}+D^{*}\right)^{2}}{D^{*} K_{D} v_{D}\left(D^{*}\left(K_{D}+K_{A}\right)+2 K_{D} K_{A}\right)} & \text { for } j_{D A}^{\text {saturating }}
\end{array} .\right.
$$

For linear control (linear $\left.j_{D A}\right), b^{-1}$ starts very high and approaches a constant as $D^{*}$ increases. We can therefore expect, as stress increases, supra-linear correlation between $D^{*}$ and $Z^{*}$ for small stressor intensities, and a constant ratio for $D^{*}$ significantly higher than $2 K_{A}$.

For saturating $j_{D A}, b^{-1}$ is again extremely large when $D^{*}$ is small (small $P_{Z}$ ), reduces for intermediate $D^{*}$, and approaches infinity as $D^{*}$ increases further. We could, therefore, expect that the correlation between $Z^{*}$ and $D^{*}$, as stress intensity increases, is first supra-linear, then sub-linear, and then supra-linear again. Consequently, the type of correlation between $Z^{*}$ and $D^{*}$ will depend on parameter values.

Next, let us consider changes in effects of increase in $P_{D}$. Since $P_{D}$ only affects the $D$ isocline, it is opportune to look at the shift of the equilibrium along the $Z$-isocline:

$$
\delta D^{*}=a \delta Z^{*} .
$$


806

$$
a=\frac{\partial D(Z)}{\partial Z}=\frac{1}{\Phi_{Z D}^{+}}\left(k_{Z}+\frac{\partial R_{Z}}{\partial Z}\right)=\frac{1}{\Phi_{Z D}^{+}} \begin{cases}k_{Z}+\frac{Z^{*} v_{Z}\left(Z^{*}+2 K_{E}\right)}{\left(K_{E}+Z^{*}\right)^{2}} & \text { for } j_{Z E}^{\text {linear }} \\ k_{Z}+\frac{Z^{*} K_{Z} v_{Z}\left(Z^{*}\left(K_{Z}+K_{E}\right)+2 K_{Z} K_{E}\right)}{\left(K_{E}+Z^{*}\right)^{2}\left(K_{Z}+Z^{*}\right)^{2}} & \text { for } j_{Z E}^{\text {saturating }}\end{cases}
$$

807 For linear $j_{Z E}$, the correlation between $Z^{*}$ and $D^{*}$ increases until it reaches a constant value. ${ }_{808}$ Therefore, we can expect sub-linear (smaller increase in $D^{*}$ than $Z^{*}$ ) correlation for small, and 809 linear correlation for large $Z^{*}$.

810 For non-linear $j_{Z E}, a$ increases with $Z^{*}$ for small values of $Z^{*}$, then decreases for intermediate ${ }_{811} Z^{*}$, and finally approaches a constant for large $Z^{*}$. Hence, conditioned on the existence of the 812 equilibrium, the type of correlation will depend on the parameters.

\section{${ }_{813}$ D.2 The ratio of $Z$ and $D$ in a runaway}

${ }_{814}$ Starting from the general dynamics (C.1), we first investigate three combinations of control types ${ }_{815}$ (see also Table 2): (1) linear $R_{Z}$ and $R_{D}$, (2) saturating $R_{Z}$ and linear $R_{D}$, and (3) linear $R_{Z}$ and 816 saturating $R_{D}$. Next, we investigate how dynamics changes when the positive feedback from $D$ to ${ }_{817} Z$ is negligible $\left(g_{Z D}=\gamma_{Z D}=0\right)$.

${ }_{818}$ Since in a runaway $Z$ and $D$ can be expected to be high enough that $Z \gg K_{E}, K_{Z}$ and ${ }_{819} D \gg K_{A}, K_{D}$, we approximate (C.1) with a non-homogeneous linear system of ODEs and recast 820 it into a matrix form:

$$
\frac{d}{d t}\left[\begin{array}{c}
Z \\
D
\end{array}\right]=\boldsymbol{A}_{\mathbf{i}}\left[\begin{array}{c}
Z \\
D
\end{array}\right]+\boldsymbol{g}_{\mathbf{i}}
$$


821

where the subscript $\mathbf{i}=1,2,3$ represents one of the three cases, and
824

825

826

Provided the matrix $A_{i}$ is not singular, and $P_{Z}$ and $P_{D}$ are constants, the particular integral of (D.5) is a constant and can be ignored for large enough $Z$ and $D$. Furthermore, only solutions to the homogeneous equation

$$
\frac{d}{d t}\left[\begin{array}{c}
Z \\
D
\end{array}\right]=\boldsymbol{A}_{\mathbf{i}}\left[\begin{array}{l}
Z \\
D
\end{array}\right]
$$

are of interest. The solutions for either state variable are a sum of two exponential functions of time $\left(a_{Z, D}^{i} e^{\lambda_{1}^{i} t}+b_{Z, D}^{i} e^{\lambda_{2}^{i} t}\right)$ where $\lambda_{1,2}^{i}$ are the eigenvalues of $\mathbf{A}_{\mathbf{i}}$, and $a_{Z, D}$ and $b_{Z, D}$ the corresponding coefficients for $Z$ and $D$. Eventually, the dominant eigenvalue ( $\lambda_{1}^{i}$ by convention) will prevail, and the ratio of $Z$ and $D$ will be constant:

$$
\lim _{t \rightarrow \infty} \frac{Z}{D}=\frac{a_{Z}^{i}}{a_{D}^{i}} .
$$

Analytic solutions are cumbersome, but solutions for specific sets of parameters can easily be calculated by solving (D.7), and the accuracy increased by taking into the account the complete solution of (D.5).

Removing the positive feedback of $D$ on $Z$ changes the runaway dynamics considerably. When $g_{Z D}=\gamma_{Z D}=0$, runaway is impossible for linear $R_{D}$ (the left-hand side of UPSE condition in Table 2 is zero). When $R_{D}$ is saturating, although $Z$ always has steady state, a runaway is inevitable whenever damage production $\left(y_{D} k_{Z} Z+P_{D}\right)$ is greater than the maximum damage repair $\left(v_{D}\right)$, i.e. 
${ }_{836}$ whenever $y_{D} k_{Z} Z+P_{D}>v_{D}$. Then, ROS remains constant at $Z^{*}=P_{Z}\left(k_{Z}+v_{Z}\right)^{-1}$, and damage ${ }_{837}$ increases linearly with the asymptotic rate of $\left(y_{D} k_{Z} Z+P_{D}-v_{D}\right)$. 